\title{
Fanout Optimization under a Submicron Transistor-Level Delay Model
}

\author{
P. Cocchini ${ }^{\dagger}$, M. Pedram ${ }^{\ddagger}$, G. Piccinini ${ }^{\dagger}$ and M. Zamboni ${ }^{\dagger}$ \\ ${ }^{\dagger}$ Dipartimento di Elettronica \\ Politecnico di Torino \\ C.so Duca degli Abruzzi 24, 10129 Torino Italy \\ ${ }^{\ddagger}$ Department of EE - Systems \\ University of Southern California \\ Los Angeles, CA 90089
}

\begin{abstract}
In this paper we present a new fanout optimization algorithm which is particularly suitable for digital circuits designed with submicron CMOS technologies. Restricting the class of fanout trees to the so-called bipolar $L T$-trees, the topology of the optimal fanout tree is found by means of a dynamic programming algorithm. The buffer selection is in turn performed by using a continuous buffer sizing technique based on a very accurate delay model especially developed for submicron CMOS processes. The fanout trees can distribute a signal with arbitrary polarity from the root of the tree to a set of sinks with arbitrary required time, required minimum signal slope, polarity and capacitive load. These trees can be constructed to maximize the required time at the root or to minimize the total buffer area under a required time constraint at the root. The performance of the algorithm shows several improvements with respect to conventional fanout optimization methods. More precisely, individual fanout trees are typically built with $60 \%$ and $10 \%$ lower area and delay, respectively, while the accuracy of calculated arrival times and signal slopes at the sinks have a typical agreement of 5\% with SPICE simulations. On the other hand, the area and delay improvements for entire circuits are $34 \%$ and $4 \%$, respectively. These results are obtained for a standard library which contains tapered and non tapered buffers with different strengths.
\end{abstract}

\section{INTRODUCTION}

During logic synthesis, several design steps are performed to translate the initial logic description into a physical net-list suitable for the final manufacturing. One of these steps, fanout optimization, is usually required after the technology mapping step where typically, for a large number of nodes in the circuit, the output signal must be propagated to several destinations (or sinks). If the necessary information for the destinations, such as capacitive load, signal polarity, and required signal propagation and transition times are specified, then it is possible to build a minimum-cost fanout tree which meets the timing constraints.

Theoretically, a fanout algorithm should be able to take advantage of the slack available at some outputs to increase the slack at the initially more critical outputs to achieve an equilibrium point where all outputs are equally critical. Conventional techniques commonly used for CMOS standard cells do not usually achieve this goal because of the discrete nature of the delay optimization they are based on. For example, the works reported in [1], [2], [3], [4], [5], [6] rely on a cell library with a finite number of available buffers. Furthermore they all use very simple delay models that severely limit their applicability especially when submicron processes are involved. On the other hand, the approach we present considerably improves these two aspects. Indeed, it is based on a continuous delay optimization technique made possible by the delay model adopted from [7] whose main features are high accuracy and independence from the technology in use.

The delay model is first applied to the creation of two numerical routines for the design of delay and area optimized CMOS tapered buffers. Then, a buffering algorithm uses them to create a fanout tree where the avail-

This research is sponsored in part by the SRC under contract no. 98-DJ-606. 
able slacks at the destinations are fully exploited to generate drivers whose delays are tailored to fit perfectly between the sink required times. Although this methodology requires that each new cell is inserted in the current library, we feel this does not constitute a limitation as all the major platforms nowadays available for the design of integrated circuits support tools for the automatic generation of cell layout (especially buffers and inverters). However, in case a library must contain a fixed number of cells and cannot be modified by the user, our technique can still be used, even though with less effectiveness, if each buffer generated by the optimization algorithm is rounded up to the closest corresponding element of that library.

In brief, the contribution of the present work can be summarized by the following points:

- An accurate technology independent submicron CMOS delay model is used for the computation of propagation times and output slopes.

- A novel continuous delay optimization technique based on speed and area optimized buffers is exploited.

- A restricted class of fanout trees, namely bipolar $L T$-trees is introduced. This class is larger than the class of $L T$-trees introduced in [5].

- A dynamic programming algorithm tree_selection, for the solution of the fanout problem is presented. Fanout trees can be found maximizing the required time of the root (while keeping the area at a minimum), or minimizing the area given a delay constraint.

- Over the restricted class of bipolar $L T$-trees, using optimized tapered buffers, the solution for fanout trees with maximum required time (keeping their area at a minimum) is found optimally in polynomial time. Notice that the general fanout optimization problem is NP-Complete [2].

- While treating sinks with different polarity simultaneously, on average the algorithm has lower complexity and better performances in terms of delay and area of the trees with respect to all other fanout optimization algorithms available in the SIS environment [8].

- We demonstrate that the use of only one algorithm for the fanout optimization of entire circuits is more effective than the approach of developing a spectrum of different algorithms.

- Constraints on the minimum signal slope required at the sinks can be given so that the gates which are connected to the fanout tree can be driven correctly.

- We provide an analysis of the effect of the discretization of the buffer library used in the optimization process on the quality of the produced fanout trees. Such an analysis identifies a discrete size library composed of only eight tapered buffers which represents the best trade-off between size of the library and quality of the fanout tree solutions.

- When using a fixed size library, the optimization methodology is capable of identifying possible deficiencies of that library providing information on the number and size of the new cells that must be included in order to reach an optimal solution. In other words, the solution can be optimized against a silicon process and not only against a library.

In Section II we give an overview of the delay model adopted in our work and introduce the routines used for the generation of the optimized buffers. In Section III we give some basic definitions and explain the buffering algorithm proposed for the solution of the fanout problem. Section IV reports an experimental analysis of the discretization of the buffer library used by our optimization algorithm and the results obtained testing the algorithm on different fanout problems extracted from common benchmark circuits. Concluding remarks are presented in Section V.

\section{DELAY OPTIMIZATION}

\section{A. Inverter Delay Model}

Since the buffering process which we perform for the generation of a fanout tree only involves CMOS tapered buffers, we are interested in modeling the behavior of their basic component, that is a static CMOS inverter whose schematic is reported in Figure 1. The delay model that we use throughout the paper is the one presented in [7]. It is composed of a set of analytical equations which model the output response of a CMOS inverter taking into 


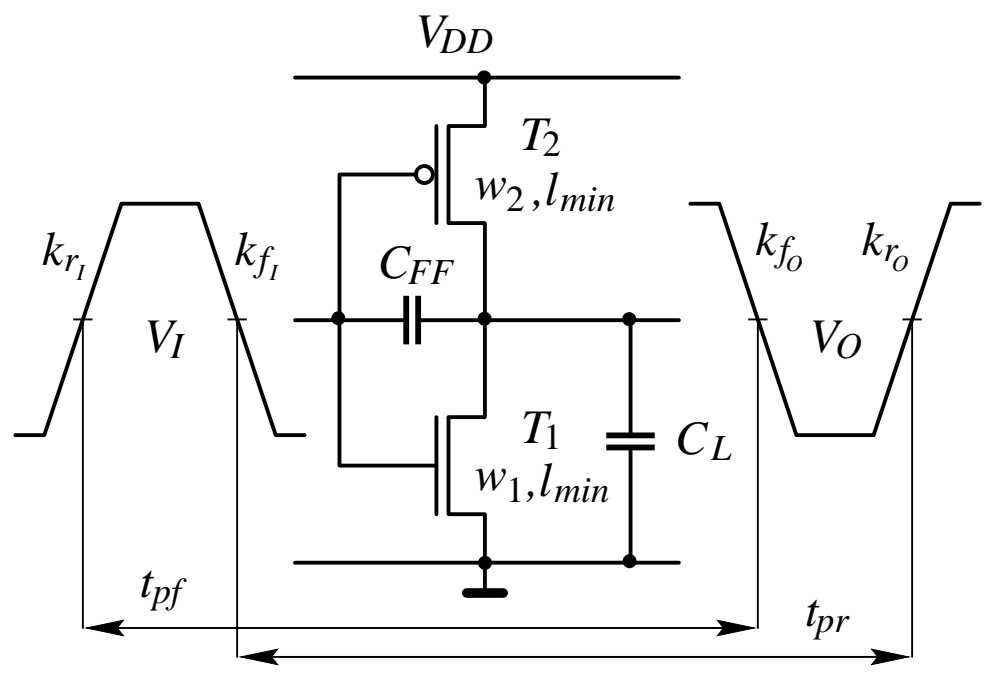

Fig. 1. CMOS Inverter.

account the main second-order effects present in submicron processes. The input voltage and output voltage are modeled as signals with trapezoidal shape as shown in Figure 1. The feed-through effect between input and output is considered by means of a capacitance $C_{F F}$. The equations depend on a small set of process parameters that can be conveniently extracted from SPICE model cards, therefore they are not tied up to any technology and do not require any calibration step. The main components of the delay mechanism are intrinsically included in the model so that the accuracy is only affected by approximations inherent to the equations. We will not give here a detailed explanation of the delay equations as this is outside the scope of the present paper. However, for a better comprehension of the work, a brief description of the mechanism with which the delay of an inverter is calculated is provided in Appendix A. For a more complete treatment, the reader is referred to [7].

In Figure 1 we also introduce some definitions used here and in the rest of the paper. With $k_{r}$ and $k_{f}$ we denote the slopes in $[\mathrm{V} / \mathrm{ns}]$ of the rising and falling edges of a ramp shape voltage signal, respectively. Following this notation, $k_{r_{I}}$ and $k_{f_{I}}$ are the slopes of the input voltage $V_{I}$ of the inverter, while $k_{r_{O}}$ and $k_{f_{O}}$ are the slopes of the output voltage $V_{O}$. Moreover, $t_{p r}$ and $t_{p f}$ are the propagation times of the rising and falling edges of $V_{O}$, respectively. They are measured as the difference between the times where $V_{O}$ and $V_{I}$ are at $50 \%$ of their total swing. An inverter $I$ is identified by the tuple $I=\{m, u\}$, where $m$ is the ratio between the width $w_{1}$ of the pull-down transistor $T_{1}$ and the minimum width $w_{\min }$ allowed by the user, and $u$ is the ratio between the widths of the pull-up and pull-down transistors of the inverter. With $P$ we denote a set of process and layout parameters of the technology in use on which the delay model depends. In this context, the equations for the delay model can be represented as:

$$
\begin{gathered}
t_{p r}=f_{1}\left(P, k_{r_{I}}, k_{f_{I}}, C_{L}, m, u\right) \\
t_{p f}=f_{2}\left(P, k_{r_{I}}, k_{f_{I}}, C_{L}, m\right) \\
k_{r_{O}}=f_{3}\left(P, k_{r_{I}}, k_{f_{I}}, C_{L}, m, u\right) \\
k_{f_{O}}=f_{4}\left(P, k_{r_{I}}, k_{f_{I}}, C_{L}, m\right)
\end{gathered}
$$

where $f_{1}, f_{2}, f_{3}, f_{4}$ are non-linear functions of their arguments (see [7] for the exact form of these functions). To automatically perform the design of an inverter and therefore of a tapered buffer, these functions have been arranged in the routine delay_INV, written in C language, which can perform two different tasks:

Task II.1: Given $P, k_{r_{I}}, k_{f_{I}}, C_{L}, m, u$, calculate $k_{r_{O}}, k_{f_{O}}, t_{p r}, t_{p f}$.

Task II.2: Given $P, k_{r_{I}}, k_{f_{I}}, C_{L}, m$, calculate $u, k_{r_{O}}, k_{f_{O}}, t_{p r}, t_{p f}$ such that $t_{p r}=t_{p f}=t_{p}$. 


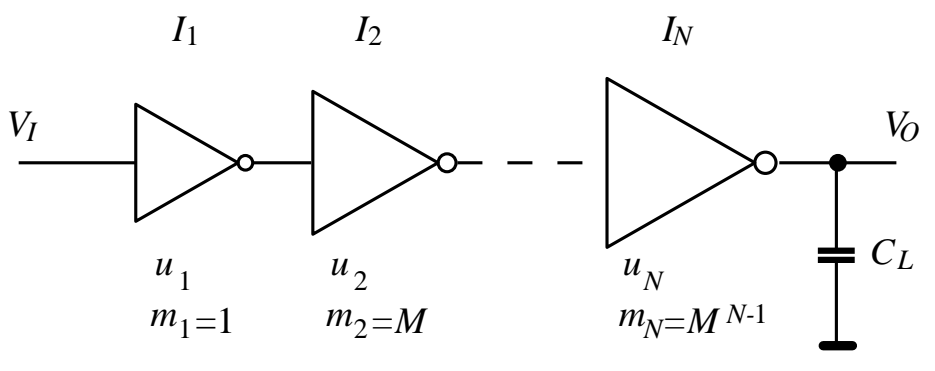

Fig. 2. CMOS Tapered Buffer.

In Task II.1, delay_INV simply computes functions $f_{1}, f_{2}, f_{3}, f_{4}$ for the given arguments. On the other hand, in Task II.2, delay_INV first solves the non linear equation

$$
f_{1}\left(P, k_{r_{I}}, k_{f_{I}}, C_{L}, m, u_{x}\right)=f_{2}\left(P, k_{r_{I}}, k_{f_{I}}, C_{L}, m\right)
$$

for the width ratio $u_{x}$ and then computes the remaining functions $f_{3}$ and $f_{4}$. Here, it must be noted that (1) is solved with very few iterations of functions $f_{1}$ and $f_{2}$ as the delay model has the ability of providing an initial value for $u$ very close to $u_{x}$.

The routine delay_INV has been applied to the calculation of the delays $t_{p r}$ and $t_{p f}$ for a minimum size inverter designed with a $0.7 \mu \mathrm{m}$ CMOS technology for a wide range of input voltage slopes and capacitive loads. While the typical agreement with SPICE simulations was 3\% in the case of task II.1 the routine was over 1000 times faster in terms of CPU time.

\section{B. Buffer Design}

A scheme of the buffers used for driving a large capacitive load is reported in Figure 2. As can be seen, the circuit is composed of a cascade of $N$ inverters each one scaled up by a factor of $M$ with respect to the previous one (the first inverter has minimum size). A buffer $B$ is then defined as a set of $N$ inverters $B=\left\{I_{1}, I_{2}, \ldots, I_{N}\right\}$. We extend here the definition of delays and signal slopes for the voltages $V_{I}$ and $V_{O}$ given in the previous section. A methodology for the determination of the optimal parameters $N$ and $M$ of a buffer with minimum and symmetrical propagation delay $\left(t_{p r}=t_{p f}=t_{p}\right)$ is given in [7]. After an initial step that characterizes a cascade of inverters with different sizes for each process in use, speed optimized tapered buffers are designed which uniformly distribute the overall propagation delay $t_{p}$ along the chain for any given capacitive load $C_{L}$. For the convenience of the reader, a short description of this methodology is provided in Appendix B.

A limitation of this buffer optimization technique is that it considers only typical values for the input slopes $k_{r_{I}}$ and $k_{f_{I}}$. Thus, to overcome this problem and consider arbitrary input slope values, the design of a minimum delay buffer is performed in this work by means of a new routine min_delay_BUF which improves the technique of [7] and that is capable of performing the following task:

Task II.3: Given $P, k_{r_{I}}, k_{f_{I}}$ and $C_{L}$, find a buffer $B$ with minimum and symmetrical propagation delay $t_{p r}=$ $t_{p f}=t_{p}$

As will be explained in Section III, such a routine is usually executed when one or more sinks in a fanout tree have to be driven introducing a delay optimized buffer. In the situation where this delay could propagate to the root of the tree, it is important to assign a buffer with the minimum possible delay $t_{p_{\min }}$ so that the required time at the source of the tree is decreased the least. Nevertheless, in many cases the slack between different buffered sinks can be of an extent such that the propagation delay $t_{p}$ can be relaxed assuming a higher value $t_{p}>t_{p_{\min }}$, so that a considerable amount of area can be saved. This situation, which recurs in most of the fanout problems commonly encountered during the automatic synthesis of digital circuits, is the key factor of the continuous delay optimization we propose, and can be exploited by means of routine min_area_BUF that performs the following task: 
Task II.4: Given $P, k_{r_{I}}, k_{f_{I}}, C_{L}, t_{p_{\text {max }}}, k_{r_{r e q}}, k_{f_{r e q}}$, find a buffer $B$ with minimum area such that $t_{p r}=t_{p f}=$ $t_{p} \leq t_{p_{\text {max }}}, k_{r_{O}} \geq k_{r_{r e q}}$ and $k_{f_{O}} \geq k_{f_{r e q}}$.

As can be seen, min_area_BUF is capable of designing a buffer with minimum area given a time constraint in terms of maximum propagation delay. Additional constraints on the minimum slope of the rising and falling edges of the output signal are also given in order not to worsen the delay of successive stages.

Both routines min_delay_BUF and min_area_BUF are based on iterative calls to routine delay_INV which is used to compute the exact delay of each stage. Thus, the propagation delays $t_{p r}$ and $t_{p f}$ and the slopes $k_{r_{O}}$ and $k_{f_{O}}$ at the output of a buffer $B$ can be put in the form

$$
\begin{gathered}
t_{p r}=f_{5}\left(P, k_{r_{I}}, k_{f_{I}}, C_{L}, N, M, u_{N}\right) \\
t_{p f}=f_{6}\left(P, k_{r_{I}}, k_{f_{I}}, C_{L}, N, M\right) \\
k_{r_{O}}=f_{7}\left(P, k_{r_{I}}, k_{f_{I}}, C_{L}, N, M, u_{N}\right) \\
k_{f_{O}}=f_{8}\left(P, k_{r_{I}}, k_{f_{I}}, C_{L}, N, M\right)
\end{gathered}
$$

where $f_{5}, f_{6}, f_{7}, f_{8}$ are non linear functions, $N$ is the the number of stages, $M$ is the tapering factor, and $u_{N}$ is the width ratio of the last inverter of buffer $B$. The values for the width ratio $u$ of all the other stages are not specified as they remain fixed to default values.

In the case of task II.3, routine min_delay_BUF simply calculates the parameters $N$ and $M$ of the minimum delay buffer $B$ according to the technique given in Appendix B, and then re-shapes its last stage solving the equation

$$
\begin{gathered}
f_{5}\left(P, k_{r_{I}}, k_{f_{I}}, C_{L}, N, M, u_{N}\right)= \\
f_{6}\left(P, k_{r_{I}}, k_{f_{I}}, C_{L}, N, M\right)
\end{gathered}
$$

for the the variable $u_{N}$, in order to have a symmetrical output response. On the other hand, routine min_delay_BUF determines the minimum number of stages $N_{\min }$ and the corresponding parameter $M$ of a tapered buffer whose propagation delays $t_{p r}$ and $t_{p f}$ are less then a given maximum value $t_{p_{\max }}$. In particular, to find a buffer with minimum area and delay $t_{p f} \leq t_{p_{\max }}$, min_delay_BUF first solves the non linear equation

$$
t_{p_{\max }}=f_{6}\left(P, k_{r_{I}}, k_{f_{I}}, C_{L}, N_{\min }, M_{\min }\right)
$$

for $M_{\min }$ such that $M_{\min } \geq 1$, and then calculates the variable $u_{N_{\min }}$, solving

$$
t_{p . f}=f_{5}\left(P, k_{r_{I}}, k_{f_{I}}, C_{L}, N_{\min }, M_{\min }, u_{N_{\min }}\right)
$$

where $t_{p f}=f_{6}\left(P, k_{r_{I}}, k_{f_{I}}, C_{L}, N_{\min }, M_{\min }\right)$, to have a symmetrical buffer output response. Finally, if the limits $k_{r_{r e q}}$ and $k_{f_{r e q}}$ on the the output slopes are specified, functions $f_{7}$ and $f_{8}$ are computed to verify that the requirements of task II.4 are met. Therefore if $k_{r_{O}} \leq k_{r_{r e q}}$ or $k_{f_{O}} \leq k_{f_{r e q}}$, the buffer with minimum area is designed solving the equations

$$
\begin{gathered}
k_{r_{r e q}}=f_{7}\left(P, k_{r_{I}}, k_{f_{I}}, C_{L}, N_{\text {min }}, M_{a}, u_{N_{\text {min }}}\right) \\
k_{f_{r e q}}=f_{8}\left(P, k_{r_{I}}, k_{f_{I}}, C_{L}, N_{\text {min }}, M_{b}\right)
\end{gathered}
$$

and taking $M_{\min }=\max \left(M_{a}, M_{b}\right)$.

Regarding the complexity of these routines, it must be pointed out that they are always capable of finding the solution to the corresponding set of equations after a small number of iterations (because every function $f_{i}$ is monotone in the variables of interest). Specifically, this efficiency is achieved because in all cases, consistent initial values are provided by the buffer optimization technique of [7]. 


\section{FANOUT OptimizATiON}

Like other proposed fanout optimizations [2] [4] [5], our methodology relies on ordering sinks by non-decreasing required time. While restricting the set of all the possible fanout trees, this assumption allowed us to develop an efficient algorithm of polynomial complexity using dynamic programming. Apart from the far more accurate delay model, our optimization technique has other important advantages. First of all, there is not a buffer selection process where trees with same topology lead to different solutions because of the several combinations of distinct buffers available in a library. As a matter of fact, given a tree topology, the extent of the slacks between distinct leaves uniquely identifies the shape and size of the needed buffers. Secondly, the treatment of sinks with different polarities is intrinsically implemented in the fanout algorithm and does not increase its complexity. Finally, the adoption of a pre-processing step, which is presented in Section III-H, can significantly reduce the number of distinct sinks to be driven so that the execution time of the algorithm is drastically shortened. In order to ease the task of describing the proposed methodology, in the following we give some definitions and formulate the fanout problems we consider in our work.

\section{A. Definitions}

We define $S$ as the set of $n$ destinations or sinks where a signal $v$, corresponding to the root of a tree, must be propagated. Each sink $s_{i} \in S$ has arbitrary polarity $p_{s_{i}} \in\{+,-\}$, capacitive load $l_{s_{i}}$ and required time $r_{s_{i}}$. Furthermore, sinks $\left\{s_{1}, s_{2}, \ldots, s_{n}\right\}$ of $S$ are ordered by increasing required time, that is, $\forall i \in[2, n-1]$, $r_{s_{i-1}} \leq r_{s_{i}} \leq r_{s_{i+1}}$. A group $G_{i, j}^{p} \subset S$ is then defined as the set of sinks of polarity $p$ among the adjacent sinks $\left\{s_{i}, \ldots, s_{j}\right\} \subset S, l_{i, j}^{p}$ being the sum of the loads of its elements. Each group $G_{i, j}^{p}$ can be driven by a corresponding buffer $B_{i}^{p}$, whose input $b_{i}^{p}$ has required time $r_{b_{i}^{p}}$ and load $l_{b_{i}^{p}}$ equal to the input capacitance of a minimum inverter, that is the one of its first stage. Finally, a fanout tree is defined as the set $T=\cup_{i} B_{i}^{p}$ of buffers $B_{i}^{p}$ that form a tree where the leaves are groups and the union of all leaves equals $S$. Under these definitions, the fanout problem can be specified in two different ways depending on the cost function to be minimized.

Problem III.1 (Max required time with Min area) Build a tree $T$ of buffers that distributes the signal $v$ to the sinks $S$ and 1) maximizes the required time $r_{v}$ at its root,2) minimizes the area of its implementation.

Problem III.2 (Min area under required time constraint) Build a fanout tree $T$ that minimizes the area of its implementation such that the required time $r_{v}$ at the root is $r_{v} \geq r_{v_{\min }}$ where $r_{v_{\min }}$ is a given minimum value. Notice that in Problem III.1 we first optimize for the maximum required time, and then minimize the area at no cost for delay. In contrast, in Problem III.2, given a minimum required time, we minimize the area subject to that constraint.

Additional constraints to these problems are the specification of a minimum signal voltage slope at the sinks as well as the minimum slopes $k_{r_{v}}$ and $k_{f_{v}}$ of the signal to be propagated.

\section{B. Tree Search Space}

In order to reduce the complexity of the algorithm only a subset of all the possible trees is considered. This subset is small enough to permit a fast generation of solutions and large enough to satisfactorily solve a large spectrum of fanout problems. A scheme representing the topology of a fanout tree belonging to such a subset is reported in Figure 3.

In this representation, sinks $S=\left\{s_{1}, s_{2}, \ldots, s_{n}\right\}$ are reported in order of increasing required time along the vertical axis, with the indication of their polarity, while buffers are drawn as small circles annotated with the number of stages they are composed of. A tree is divided into a set of $z$ different levels identified by a $(\mathrm{z}+1)$ tuple of integers $\left(y_{1}, \ldots, y_{z+1}\right)$ such that: $y_{1}=1<y_{2}<\ldots<y_{z}<y_{z+1}=n+1$, with $1 \leq z \leq n$. Each level $i \in\{1, \ldots, z\}$ contains $y_{i+1}-y_{i}$ sinks, from $s_{y_{i}}$ to $s_{y_{i+1}-1}$. Sinks with positive polarity form the group $G_{y_{i}, y_{i+1}-1}^{+}$and are driven by a buffer $B_{y_{i}}^{+}$whereas those with negative polarity form the group $G_{y_{i}, y_{i+1}-1}^{-}$and are driven by a buffer $B_{y_{i}}^{-}$. In the case of Figure $3, z=3$ with a $(\mathrm{z}+1)$-tuple $(1,3,9,17)$. Each buffer can accept 


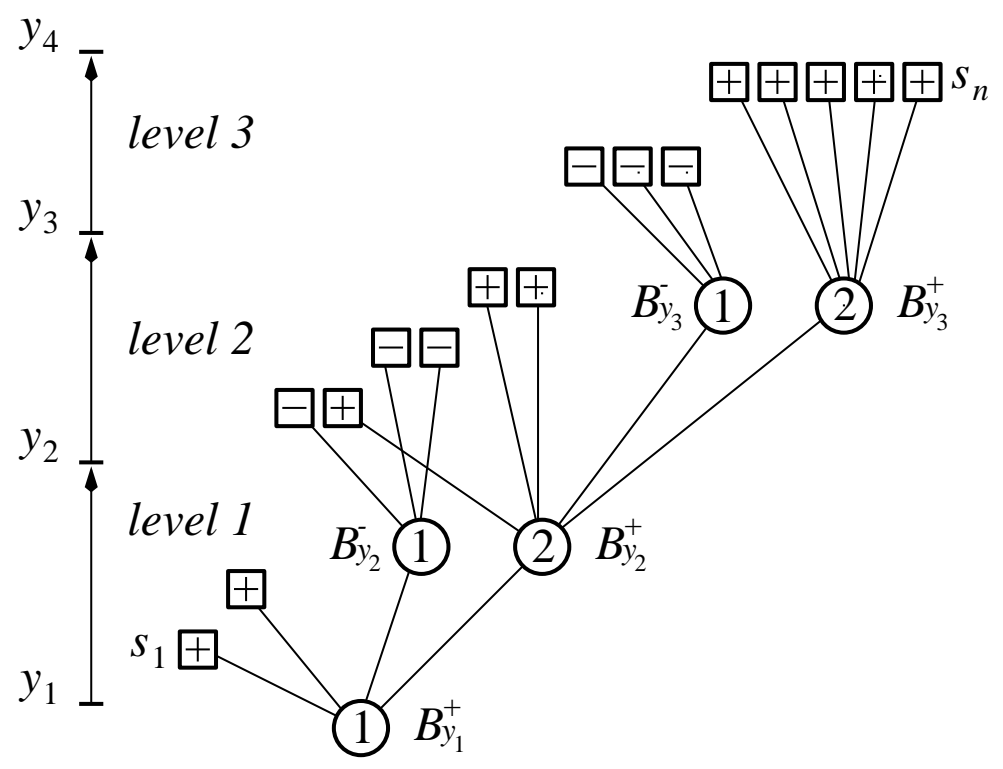

Fig. 3. Example of tree topology with three distinct levels.

a connection from one or two buffers belonging to the upper level $i+1$. Depending on the polarities of its sinks and the buffers of the upper level $i+1$, it follows that a level $i$ can always have exactly one or two buffers driving its sinks.

The class of trees that we have just defined is very similar to that of $L T$-Trees of type 1 introduced in [5]. While the trees belonging to such class have at most one buffer in the fanout of any buffer, in our case each buffer can drive 1 or 2 buffers along with any number of leaves. For this reason, we call our trees bipolar $L T$-Trees, or shortly Bi- $L T$-Trees.

Because of this property, it is apparent that each $(z+1)$-tuple identifies $2^{z}$ possible fanout trees. The number of possible $(\mathrm{z}+1)$-tuples of integers corresponds to the number of distinct ways of choosing $z-1$ elements among $n-1$. Therefore, the total number of possible fanout trees is

$$
\begin{gathered}
\sum_{z=1}^{n-1}\left(\begin{array}{c}
n-1 \\
z-1
\end{array}\right) 2^{z}=2 \sum_{z=0}^{n-2}\left(\begin{array}{c}
n-1 \\
z
\end{array}\right) 2^{z}= \\
2 \cdot 3^{n-1}-2^{n}
\end{gathered}
$$

Such search space is greater than both that of $L T$-Trees of type $1\left(2^{n-2}\right)$, and $L T$-Trees of type $2\left(2^{n-1}\right)$ [5].

In (7) we also assume that the first level can have two buffers which are driving sinks of different polarities. It is apparent that this situation is in contrast with the requirement of a one-rooted fanout tree like the one of Figure 3. Nevertheless, every occurrence of this kind can be uniquely resolved introducing one or two additional inverters in case $p_{b_{1}^{+}} \cdot p_{b_{1}^{-}}=+$or $p_{b_{1}^{+}} \cdot p_{b_{1}^{-}}=-$, respectively, so that the equation still holds.

\section{The Algorithm for Tree Selection}

The selection of the best tree for the solution of Problems III.1 and III.2 is performed with the algorithm tree_selection, detailed in Figure 4. At the beginning, the process database $P$ is loaded and sinks are ordered by non-decreasing required time. Then, the load $l_{i, j}^{p}$ of each possible group $G_{i, j}^{p} \subset S$ is pre-computed. The problem is now split in $n$ sub-problems, identified by an index $z$, of sinks $\left(s_{z}, \cdots, s_{n}\right)$. A sub-problem $z$, then, is solved in $n-z+1$ different ways, indicated by an index $h$, of which only the best one $T_{z}$ is kept in a table, hence this is a dynamic programming approach. Each solution $h$ corresponds to the insertion of one or two 
buffers $B^{+}$and/or $B^{-}$, which respectively drive groups $G_{z, h}^{+}$and $G_{z, h}^{-}$, and the upper level sub-tree $T_{h+1}$. Since the algorithm proceeds with $z$ from $n$ to $1, T_{h+1}$ has already been computed and is available.

For each polarity $p \in\{+,-\}$, the load of a buffer $B^{p}$ is calculated as the sum of the pre-computed quantity $l_{z, h}^{p}$

and the load of same polarity $l_{T_{h+1}}^{p}$, offered by the sub-tree $T_{h+1}$. If such load is null, the corresponding buffer $B^{p}$ is not inserted. Each buffer is designed calling the routine min_area BUF whose arguments are ordered, and have the same meaning, as in the definition of task II.4. Particularly, the slopes $k_{r}$ and $k_{f}$ of the input signal are chosen as typical values for a correct execution of the algorithm.

As can be seen, the maximum allowed delay time $t_{\max }=r_{\text {load }}-r_{\text {prev }}$ is equal to the difference of two terms: the required time $r_{\text {load }}$ of the load driven by the buffer, and $r_{p r e v}$ which is equal to the required time $r_{s_{z-1}}$ of the closest not yet buffered sink $s_{z-1}$. In this way, buffer $B^{p}$ will have a required time equal or higher than $r_{s_{z-1}}$, thus not affecting the required time of subsequent sub-trees, and minimum area for its implementation. If $t_{\max }$ is too low and no buffer with such delay is possible, then $B^{p}$ is designed by means of routine min_delay_BUF, which, given its arguments defined as for task II.3, returns a minimum delay buffer. At this point, the $h$ solution $T$ of sub-problem $z$ is formed by the union of buffers $B^{+}, B^{-}$and sub-tree $T_{h+1}$.

If the required time $r_{T}$ of $T$, defined as the minimum of the required times of $B^{+}$and $B^{-}$(or the required time of one of them if the other is empty), is higher than $t_{\text {prev }}$, and its area is lower than the one of the best current solution $T_{z}$, then sub-tree $T$ takes its place. On the other hand, if $r_{T}$ is lower than $t_{p r e v}, T$ is stored only if its required time is the highest.

The same procedure applies to both Problems III.1 and III.2 until the last sub-problem $z=1$, which corresponds to the overall fanout problem, has to be solved. As can be seen, in such a situation the required time $t_{\text {prev }}$ takes different values. When Problem III.1 is being solved, then $r_{\text {prev }}=r_{\text {load }}$ and buffers $B^{p}$ are designed for minimum delay. On the other hand, for Problem III.2, $r_{p r e v}$ takes the value $r_{v_{\text {min }}}$, the given minimum required time of the root that can be exploited by the routine min_area_BUF to obtain a buffer with lower area. In this way, at the end of the process, tree $T_{1}$ stores the best solution for a given fanout problem.

\section{Optimality for the Minimum Delay Problem}

The optimality of the algorithm for the solution of Problem III.1 is proved by the following theorem:

Theorem III.1: The tree_selection algorithm produces an optimal fanout tree for Problem III.1 over the class of all Bi-LT-Trees, assuming that routine min_delay_BUF produces optimal solutions to task II.3.

Proof: From the property of dynamic programming algorithms, the solution to Problem III.1 is optimal exactly if such is true for the solution $T_{z}$ of each sub-problem $z$. Therefore, for what pertains to the proof of the theorem, it is sufficient to prove the optimality of a single sub-tree $T_{z}$. The rest of the proof follows by induction on $z$.

The solution of a sub-problem $z$, takes the generation of $n-z+1$ different sub-trees by means of routines min_delay_BUF and min_area_BUF. In each case, min_area_BUF introduces a buffer whose required time is always greater than the required time $r_{p r e v}$ of the highest sink in the lower level. On the other hand, min_delay_BUF generates a speed optimized buffer whose delay is the smallest possible. The solution $T_{z}$ is then chosen as the one with the highest required time $r_{T}$ if every sub-solution has required time $r_{T}<r_{\text {prev }}$; otherwise the sub-tree with minimum area is taken. As a result, the solution $T_{z}$ is optimal because it will offer to the next sub-problem $z-1$, the smallest load to drive (the input capacitance of a buffer is always that of a minimum size inverter), with a required time such that the required time $r_{T_{z-1}}$ of the root of the subsequent sub-tree $T_{z-1}$ can be the maximum possible.

\section{E. Optimality for the Minimum Area Problem}

Unlike the case of Problem III.1, the tree_selection algorithm does not produce the optimal solution to Problem III.2. Nevertheless, this shortcoming can be easily remedied by adopting a binning technique (similarly 


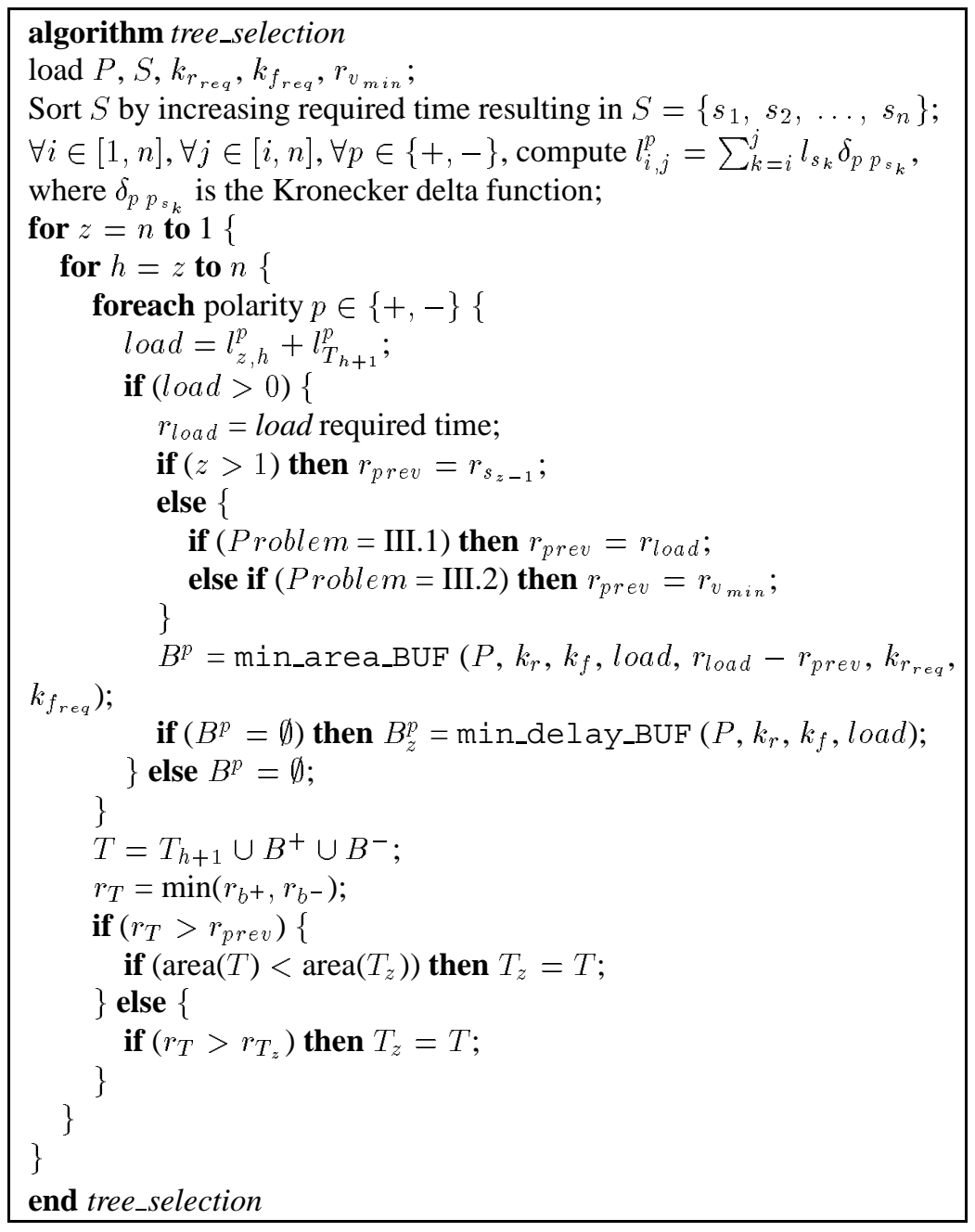

Fig. 4. The algorithm for the fanout tree selection.

to the one used in [5]), modifying the algorithm at the cost of increased complexity. More precisely, for each tuple $(z, h)$ such that $z>1$, the minimum required time $r_{p r e v}$ given as argument to the routine min_area_BUF has to assume a discrete set of $\tau$ different possible values. Therefore, each sub-problem $z$ results in a richer spectrum of $\tau(n-z+1)$ sub-solutions $T$, each one with different area and required time, available for the generation of the fanout tree. In this way, neglecting the error introduced by the discretization of $r_{\text {prev }}$, the optimality for the solution to Problem III.2 is achieved with complexity $O\left(\tau n^{2}\right)$. In practice, since we have found that this technique, even though it produces the optimal solution for Problem III.2, does not provide substantial area improvements compared to the basic algorithm, we have chosen to exclude it from the tree_selection algorithm in order to retain a low complexity.

\section{F. An Example of Generated Tree}

An example of a bipolar $L T$-tree generated by the algorithm tree_selection is reported in Figure 5 for a typical problem with 18 sinks and a $0.5 \mu \mathrm{m}$ CMOS process. Here, sinks and buffers are represented using the notation of Section III-B also adopted in Figure 3.

As can be seen, there are three levels. Level 1 is composed of sinks $s_{1}, s_{2}, s_{3}$ and the inverter $B_{1}^{+}$, whereas level 2 is composed of sinks $s_{4}, s_{5}$ and the inverter $B_{4}^{-}$, and level 3 is composed of sinks $s_{6}$ through $s_{18}$ and 


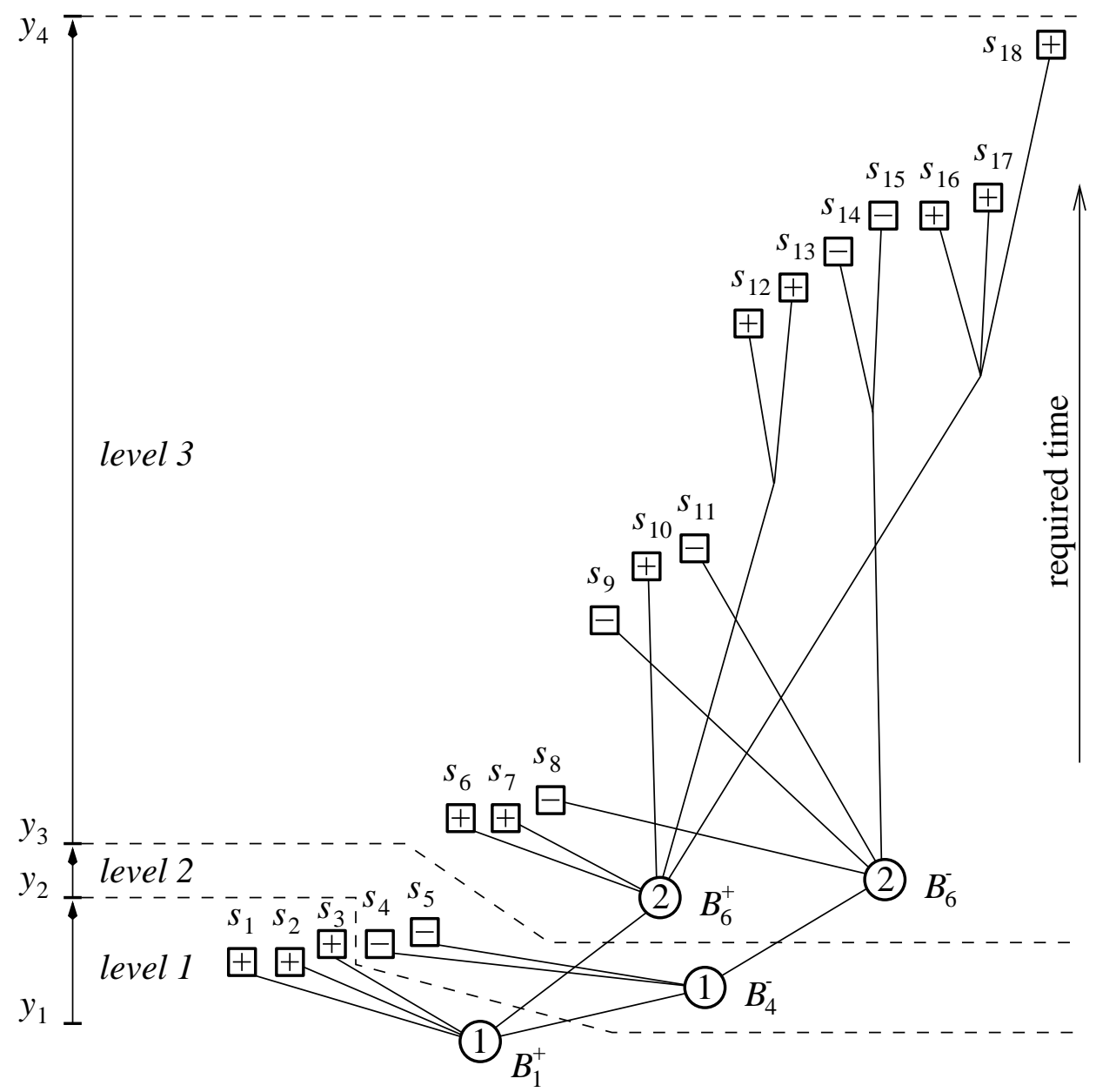

Fig. 5. Bipolar $L T$-tree for a typical problem: $n=18, z=3, y_{1}=1, y_{2}=4, y_{3}=6, y_{4}=18$.

the two-stage buffers $B_{6}^{+}$and $B_{6}^{-}$. It is interesting to note that the required time at both buffers $B_{6}^{+}$and $B_{6}^{-}$is greater than that at any of the sinks belonging to the lower level $2\left(B_{6}^{+}\right.$and $B_{6}^{-}$have both higher position than sinks $s_{4}$ and $s_{5}$ along the vertical direction). A thorough examination of the tree generation process indicates that both buffers have been designed to have minimum area through the routine min_area_BUF, and that the minimization process stopped because of the constraints on the minimum slopes $k_{r_{r e q}}, k_{f_{r e q}}$ of the output signal. This situation, which is very recurrent in almost all of the standard-cell based fanout problems, is fully exploited by our buffering mechanism, leading to the generation of a fanout tree with the lowest area cost.

\section{G. Complexity}

The number of times we go through the most nested inner loop of the tree_selection algorithm is equal to $n(n+1)$. Therefore the complexity of the algorithm is $O\left(n^{2}\right)$, as we assume that both routines min_delay_BUF and min_area_BUF have complexity $O(1)$ and perform their respective tasks in constant time (the typical execution time of these routines are given at the end of Section IV-B). When treating sinks of different polarities simultaneously, the algorithm proposed in [5] has complexity $O\left(d^{2} \max (n, p) \max \left(n p\right.\right.$, max $\left.\left.(n, p)^{1.5}\right)\right)$, while the one proposed in [4] has complexity $O\left(d^{3} n^{2} p^{2}\right)$. Here, $d$ is the number of different buffers in the cell library, and $n$ and $p$ are the number of sinks of negative and positive polarity, respectively. As can be seen, our algorithm has smaller complexity due to the direct selection of buffers in the chosen trees. 


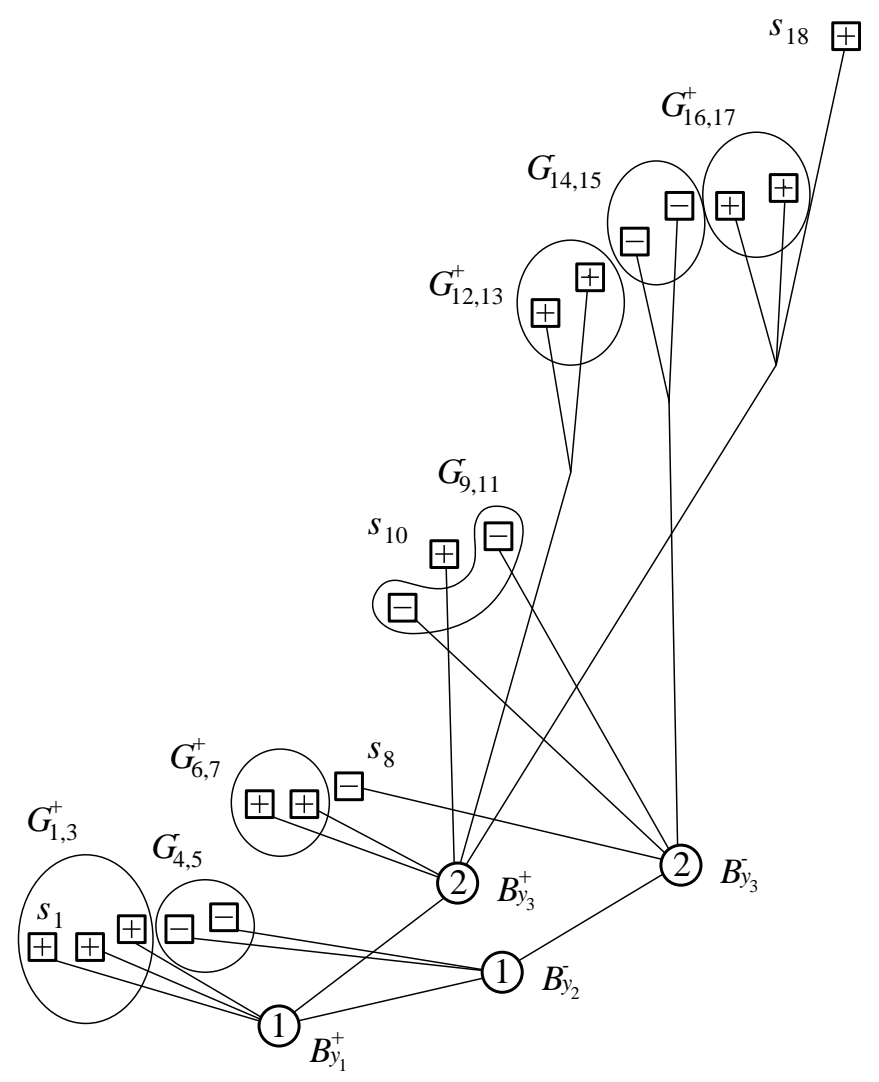

Fig. 6. Fanout tree for a typical problem with the merge_sinks pre-processing step.

\section{H. Pre-Processing}

With our methodology sinks are treated independently of their load and there are no limits imposed on their size. This property suggests that sinks with equal or very close required time can be merged together to reduce the size of the problem with no adverse impact on the final result. An example of application of this technique to the test case of Figure 5, by means of the routine merge_sinks, is shown in Figure 6. Here, the number of distinct sinks $n$ is now reduced to 10,7 of them corresponding to groups $G_{i, j}$ of sinks of the same polarity. As can be seen, the result in terms of speed and area of the fanout tree is the same as Figure 5. However, in this case the user CPU time needed by the computation is significantly lower. Since this technique makes a great improvement in the computation time of the algorithm at no performance cost, it is always used during a pre-processing step to reduce the number of distinct sinks of a fanout problem.

\section{Post-Processing}

It has already been pointed out that during the execution of the algorithm tree_selection, the slopes $k_{r}$ and $k_{f}$ of the buffer input signal are chosen as typical values. This introduces some error, although small. After the algorithm has completed its execution and the topology of the best tree is available, the delay and slopes of all the buffers of the tree can be recomputed, yielding exact values, traversing the tree from root up. Particularly, the output slopes $k_{r_{O}}, k_{f_{O}}$ of the level 1 buffers are first calculated with the given input values $k_{r_{I}}$ and $k_{f_{I}}$ of Problems III.1 and III.2, and then reused as input values for the buffers of level 2. Iterating this process for the rest of the levels, the timing of the signal $v$ distributed along the tree can then be accurately recomputed for all the intermediate nodes and destinations. 


\section{RESUlts AND VERIFICATION}

\section{A. Discrete Size Buffer Library}

We have already mentioned that our fanout optimization methodology relies on the availability of an arbitrary number of continuously sized tapered buffers for the construction of optimal fanout trees. Nevertheless, in many situations it is impractical to insert an unlimited number of new custom buffers into a cell library. Therefore, it is mandatory to restrict the number of the buffers available to the optimization process only to those included in a predefined discrete size buffer library. Every buffer generated by the optimization algorithm will be then rounded up to the closest element in that library. It is apparent that the optimality of the solutions will be affected by the size and granularity of such a library. Therefore it is of primary importance that the library to be chosen to work with the optimization algorithm meets the following two requirements:

- It contains a spectrum of buffers whose size is properly distributed in the range of values from a minimum (the size of a minimum inverter) to a maximum limit (the size of a buffer with $N_{\max }$ number of stages and $M_{\max }$ stage ratio). Such a discrete range is representative of the continuous buffer design space the optimization algorithm is based on.

- The quality of the solutions, when moving from the continuous search space to such a discrete library, is not substantially affected.

In this section we focus on the determination of such a discrete library. To do so we have applied our optimization algorithm to the solution of several fanout problems extracted from a set of six ISCAS benchmark circuits using four different $0.5 \mu \mathrm{m}$ CMOS process buffer libraries: lib-20, lib-12, lib-8, lib-4, containing 20, 12, 8, 4 tapered buffers respectively, and calibrated in DSMLib (Deep Sub-Micron library) format. In this format, the delay of each pin of each cell is characterized by four subsets of 4 parameters each, modeling the propagation times $t_{p r}$ and $t_{p f}$, and the transition times $t_{t r}$ and $t_{t f}$. The transition times $t_{t r}$ and $t_{t f}$ are here defined as the difference between the times where the rising and falling edges of a signal are at $10 \%$ and $90 \%$ of their total swing, respectively. The pin-dependent delay model is as follows:

$$
\text { delay }=\left(K_{1}+K_{2} \cdot \text { load }\right) \cdot \text { transition_time }+K_{3} \cdot \text { load }+K_{4}
$$

where delay represents any of the terms $t_{p r}, t_{p f}, t_{t r}$ and $t_{t f}$ for the output pin, load denotes the capacitive load of the cell, and transition_time refers to $t_{t r}$ or $t_{t f}$ for the input pin as appropriate. The choice of such a format has been dictated by the need for an accurate delay model which includes the effect of the slope of the voltage signals in the calculation of the standard cell timing. Notice that this delay model has only been used for the computation of the propagation and transition times during the timing analysis executed in the SIS environment ${ }^{1}$, while the actual optimization has been performed with the far more accurate delay model introduced in Section II. The composition of these libraries is reported in Table I. Here, the first column reports buffer names while the second column reports the area of their layout implementation in $\mu \mathrm{m}^{2}$. The third column reports the number of stages $N$ of each buffer, and the fourth column reports the stage ratio $M$. In the remaining columns, one for each discrete library, a dot is present if the buffer corresponding to the same row is part of that library. The name of each buffer is indicative of the number and size of the stages it is composed of. For instance, $1 \mathrm{x}$ is a minimum inverter $(N=1, M=1), 1 \mathrm{x} 1 \mathrm{x}$ is a two stage buffer with both stages of minimum size, and $1 \times 4 \times 44^{2} \mathrm{x}$ is a three stage buffer where the first stage has minimum size, the second is four times larger and the third sixteen times larger. In some cases (buffers $1 \times 2 x$ through $1 \times 3.5 x$ ), the area of buffers with adjacent stage ratio values is the same. This is because, since the height of the cell layout is fixed and the pull-up and pull-down transistors of each stage of the buffers are folded, the area of the buffer cell only increases by discrete quantities when the size of the transistors plus the needed spacing between them exceeds the height of the cell.

In the table, cell $1 \mathrm{x}$, which is a minimum inverter, is not included in the buffer count even though it is part of each library. As can be seen, lib-20 includes all of the buffers reported in the table. Here, the range of $N$ and

\footnotetext{
${ }^{1}$ In fact, here the concept of transition time is not contemplated at all.
} 


\begin{tabular}{||l|c|c|c||c|c|c|c||}
\hline \hline buffer & area & $N$ & $M$ & lib-20 & lib-12 & lib-8 & lib-4 \\
\hline \hline $1 \mathrm{x}$ & 687.5 & 1 & 1.0 & $\bullet$ & $\bullet$ & $\bullet$ & $\bullet$ \\
\hline \hline $1 \times 1 \times$ & 852.5 & 2 & 1.0 & $\bullet$ & $\bullet$ & $\bullet$ & $\bullet$ \\
\hline $1 \times 2 \times$ & 935.0 & 2 & 2.0 & $\bullet$ & $\bullet$ & $\bullet$ & \\
\hline $1 \times 2.5 \mathrm{x}$ & 935.0 & 2 & 2.5 & $\bullet$ & & & \\
\hline $1 \times 3 \mathrm{x}$ & 935.0 & 2 & 3.0 & $\bullet$ & $\bullet$ & & \\
\hline $1 \times 3.5 \mathrm{x}$ & 935.0 & 2 & 3.5 & $\bullet$ & & & \\
\hline $1 \times 4 \times$ & 1017.5 & 2 & 4.0 & $\bullet$ & $\bullet$ & $\bullet$ & \\
\hline $1 \times 4.5 \mathrm{x}$ & 1017.5 & 2 & 4.5 & $\bullet$ & & & \\
\hline $1 \times 5 \times$ & 1017.5 & 2 & 5.0 & $\bullet$ & $\bullet$ & & \\
\hline $1 \times 5.5 \mathrm{x}$ & 1017.5 & 2 & 5.5 & $\bullet$ & & & \\
\hline $1 \times 6 \times$ & 1100.0 & 2 & 6.0 & $\bullet$ & $\bullet$ & $\bullet$ & $\bullet$ \\
\hline $1 \times 2 \times 2^{2} \times$ & 1265.0 & 3 & 2.0 & $\bullet$ & $\bullet$ & $\bullet$ & \\
\hline $1 \times 2.5 \times 2.5^{2} \times$ & 1347.5 & 3 & 2.5 & $\bullet$ & & & \\
\hline $1 \times 3 \times 3^{2} \times$ & 1430.0 & 3 & 3.0 & $\bullet$ & $\bullet$ & & \\
\hline $1 \times 3.5 \times 3.5^{2} \times$ & 1595.0 & 3 & 3.5 & $\bullet$ & & & \\
\hline $1 \times 4 \times 44^{2} \times$ & 1842.5 & 3 & 4.0 & $\bullet$ & $\bullet$ & $\bullet$ & $\bullet$ \\
\hline $1 \times 2 \times 2^{2} \times 2^{3} \times$ & 1760.0 & 4 & 2.0 & $\bullet$ & $\bullet$ & $\bullet$ & \\
\hline $1 \times 2.5 \times 2.5^{2} \times 2.5^{3} \times$ & 2090.0 & 4 & 2.5 & $\bullet$ & & & \\
\hline $1 \times 3 \times 3^{2} \times 3^{3} \times$ & 2667.5 & 4 & 3.0 & $\bullet$ & $\bullet$ & & \\
\hline $1 \times 3.5 \times 3.5^{2} \times 3.5^{3} \times$ & 3492.5 & 4 & 3.5 & $\bullet$ & & & \\
\hline $1 \times 4 \times 4^{2} \times 4^{3} \times$ & 4647.5 & 4 & 4.0 & $\bullet$ & $\bullet$ & $\bullet$ & $\bullet$ \\
\hline \hline & & & & & & \\
\hline
\end{tabular}

TABLE I

COMPOSITION OF THE DISCRETE SIZE BUFFER LIBRARIES USED IN THE DISCRETIZATION ANALYSIS: AREA IS THE CELl LAYOUT AREA IN $\mu \mathrm{m}^{2}, N$ IS THE NUMBER OF STAGES AND $M$ IS THE STAGE RATIO.

$M$ has been determined by running the optimization algorithm for the benchmark circuits of Section IV-C and collecting the necessary information after the generation of the continuously sized tapered buffers. Specifically the maximum number of stages $N$ is 4 , while the stage ratio $M$ goes from 1 to 6 for two stage buffers, and from 2 to 4 for three and four stage buffers. It must be noted here, that we have chosen lib-20 as the maximum size discrete library for our analysis because running the optimization algorithm with more available buffers than those in lib-20 never produced better results. Thus, starting from library lib-20, libraries lib-12, lib-8, and lib-4 gradually decrease the granularity of the stage ratio $M$ while still covering the corresponding range of variation.

For each of the six ISCAS benchmark circuits, logic synthesis and minimum delay technology mapping steps have been performed in the SIS environment, using a standard $0.5 \mu \mathrm{m}$ CMOS technology library also calibrated in DSMlib format for the same $0.5 \mu \mathrm{m}$ CMOS process and standard cell layout style of libraries lib-20, lib-12, lib-8, and lib-4. From each circuit, four fanout problems of different complexity have been extracted and written in blif format for testing the algorithm and comparing its performance when using each of the four buffer libraries lib-20, lib-12, lib-8, and lib-4. The results for the optimized problems are reported in Table II. Here, the name of each problem and its complexity given in terms of the number $n$ of sinks are reported in the first and the second column respectively. In the lib-20 field, the results of the optimization obtained rounding up the continuously sized buffers to the elements of library lib-20 are reported. Here, area and delay are respectively the area in $\mu \mathrm{m}^{2}$ of the tree implementation and the difference in nanoseconds between the required time at the most critical 


\begin{tabular}{|c|c|c|c|c|c|c|c|c|c|c|c|c|c|c|c|}
\hline \multicolumn{2}{|c|}{ problem } & \multicolumn{2}{|c|}{ lib-20 } & \multicolumn{4}{|c|}{ lib-12 } & \multicolumn{4}{|c|}{ lib-8 } & \multicolumn{4}{|c|}{ lib-4 } \\
\hline name & $n$ & area & delay & area & $\Delta \%$ & delay & $\Delta \%$ & area & $\Delta \%$ & delay & $\Delta \%$ & area & $\Delta \%$ & delay & $\Delta \%$ \\
\hline $\bar{C} 1355-1$ & $\overline{8}$ & 3328 & 0.44 & 3410 & 2.5 & $\overline{0.44}$ & $\overline{0.0}$ & 3410 & 2.5 & $\overline{0.44}$ & $\overline{0.0}$ & 3492 & 4.9 & $\overline{0.44}$ & $\overline{0.0}$ \\
\hline C1355-2 & 9 & $\overline{932}$ & 0.47 & 3932 & 0.0 & 0.47 & 0.0 & 3932 & 0.0 & 0.47 & 0.0 & 4015 & 2.1 & 0.51 & 8.5 \\
\hline C1355-3 & 9 & 2392 & 0.52 & 2392 & 0.0 & 0.52 & 0.0 & 3740 & 56.4 & 0.53 & 1.9 & 2475 & 3.5 & 0.54 & 3.8 \\
\hline C1355-4 & 13 & 1018 & 0.36 & 1018 & 0.0 & 0.36 & 0.0 & 1018 & 0.0 & 0.36 & 0.0 & 1100 & 8.1 & 0.50 & 38.9 \\
\hline C3540-1 & 12 & 4455 & 0.41 & 4455 & $\overline{0.0}$ & 0.41 & $\overline{0.0}$ & 4455 & $\overline{0.0}$ & 0.41 & 0.0 & 4455 & $\overline{0.0}$ & 0.41 & 0.0 \\
\hline C3540-2 & 35 & 4042 & 0.24 & 4620 & 14.3 & 0.24 & 0.0 & 6600 & 63.3 & 0.24 & 0.0 & 6600 & 63.3 & 0.24 & 0.0 \\
\hline C3540-3 & 21 & 3575 & 0.48 & 3575 & 0.0 & 0.48 & $\overline{0.0}$ & 3740 & 4.6 & 0.50 & 4.2 & 3740 & 4.6 & 0.50 & 4.2 \\
\hline C3540-4 & 72 & 6352 & 0.19 & 6518 & 2.6 & 0.19 & 0.0 & 7012 & 10.4 & 0.19 & 0.0 & 7095 & 11.7 & 0.19 & 0.0 \\
\hline C432-1 & 16 & 1952 & 0.23 & 1952 & 0.0 & 0.23 & 0.0 & 1952 & 0.0 & 0.23 & 0.0 & 1952 & 0.0 & 0.23 & 0.0 \\
\hline $\mathrm{C} 432-2$ & 6 & 852 & 0.21 & 852 & 0.0 & 0.21 & 0.0 & 852 & 0.0 & 0.21 & 0.0 & 852 & 0.0 & 0.21 & 0.0 \\
\hline C432-3 & 16 & 2805 & 0.30 & 2805 & 0.0 & 0.30 & $\overline{0.0}$ & 2888 & 3.0 & 0.30 & 0.0 & 2888 & 3.0 & 0.30 & $\overline{0.0}$ \\
\hline C432-4 & 10 & 2558 & 0.16 & 2558 & 0.0 & 0.16 & $\overline{0.0}$ & 2558 & $\overline{0.0}$ & 0.16 & 0.0 & 2640 & 3.2 & 0.16 & $\overline{0.0}$ \\
\hline C5315-1 & 49 & $\longdiv { 3 6 8 }$ & 0.49 & 10532 & 1.6 & 0.49 & 0.0 & 10532 & 1.6 & 0.49 & 0.0 & 10945 & 5.6 & 0.52 & 6.1 \\
\hline C5315-2 & 12 & 3080 & 0.30 & 3080 & 0.0 & 0.30 & $\overline{0.0}$ & 3080 & 0.0 & 0.30 & 0.0 & 3080 & $\overline{0.0}$ & 0.30 & $\overline{0.0}$ \\
\hline C5315-3 & 21 & 7178 & 0.40 & 7178 & 0.0 & 0.40 & 0.0 & 7178 & 0.0 & 0.40 & 0.0 & 7342 & 2.3 & 0.44 & 10.0 \\
\hline C5315-4 & 50 & 10120 & 0.51 & 10285 & 1.6 & 0.52 & 2.0 & 10450 & 3.3 & 0.52 & 2.0 & 10615 & 4.9 & 0.52 & 2.0 \\
\hline C6288-1 & 16 & 1100 & 0.17 & 1100 & 0.0 & 0.17 & 0.0 & 1100 & 0.0 & 0.17 & 0.0 & 1100 & 0.0 & 0.17 & $\overline{0.0}$ \\
\hline C6288-2 & 21 & 1100 & 0.17 & 1100 & 0.0 & 0.17 & $\overline{0.0}$ & 1100 & 0.0 & 0.17 & 0.0 & 1100 & 0.0 & 0.17 & $\overline{0.0}$ \\
\hline C6288-3 & 60 & 8690 & 0.29 & 8772 & 0.9 & 0.29 & $\overline{0.0}$ & 8938 & 2.9 & 0.29 & 0.0 & 9955 & 14.6 & 0.44 & 51.7 \\
\hline C6288-4 & 50 & 1100 & 0.17 & 1100 & 0.0 & 0.17 & 0.0 & 1100 & 0.0 & 0.17 & 0.0 & 1100 & 0.0 & 0.17 & 0.0 \\
\hline C7552-1 & 283 & 38775 & 0.52 & 39682 & 2.3 & 0.53 & 1.9 & 38802 & 0.1 & 0.56 & 7.7 & 38802 & 0.1 & 0.56 & 7.7 \\
\hline C7552-2 & 12 & 1705 & 0.26 & 1705 & 0.0 & 0.26 & 0.0 & 1788 & 4.9 & 0.26 & 0.0 & 1788 & 4.9 & 0.26 & 0.0 \\
\hline C7552-3 & 16 & 4620 & 0.23 & 4620 & 0.0 & 0.23 & 0.0 & 4620 & 0.0 & 0.23 & 0.0 & 4620 & 0.0 & 0.23 & $\overline{0.0}$ \\
\hline C7552-4 & 23 & 1788 & 0.18 & 1788 & 0.0 & 0.18 & 0.0 & 1788 & 0.0 & 0.18 & 0.0 & 1788 & 0.0 & 0.18 & $\overline{0.0}$ \\
\hline average & & & & & 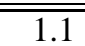 & & $\overline{0.2}$ & & 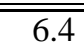 & & 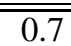 & & "5.7 & & $\overline{\overline{5.5}}$ \\
\hline
\end{tabular}

TABLE II

RESULTS FOR DELAY OPTIMIZED FANOUT TREES USING FOUR DIFFERENT DISCRETE SIZE BUFFER LIBRARIES. FOR EACH TREE, DELAY IS THE DIFFERENCE IN NANOSECONDS BETWEEN THE REQUIRED TIME AT THE MOST CRITICAL SINK AND THE REQUIRED TIME AT THE ROOT DRIVER, AND AREA IS THE AREA IN $\mu \mathrm{m}^{2}$ OF THE TREE IMPLEMENTATION.

sink $s_{1}$ (the first one since sinks are sorted in order of non-decreasing required time) and the required time at the driver of the root of the tree. The same results for area and delay are then reported for libraries lib-12, lib-8, and lib-4 in the corresponding fields. Furthermore, in these last three fields, to the right of each area and delay column, another column indicated by $\Delta \%$ reports the percentage variation of the values to its left with respect to the corresponding values in the lib-20 field. For example, problem C5315-4 has area equal to $10285 \mu \mathrm{m}^{2}$ and delay equal to $0.52 \mathrm{~ns}$ when using library lib-12, these values being respectively $1.6 \%$ and $2 \%$ higher than those corresponding to library lib-20, that is area $=10120 \mu \mathrm{m}^{2}$ and delay $=0.51 \mathrm{~ns}$.

As can be seen, for several problems (e.g. C3540-1) there are no variations in area and delay $(\Delta \%=0)$ when going from the lib-20 field to the lib-12, lib-8, and lib-4 fields. As a matter of fact, in all of these cases the fanout trees are only composed of buffers which are present in all four libraries (typically only minimum inverters $1 \mathrm{x}$ and minimum size buffers $1 \mathrm{x} 1 \mathrm{x})$.

In other problems (e.g. C3540-2 when going from lib-20 to lib-12) when shifting to a smaller size discrete library, only the area increases while the delay remains the same. The reason why this happens is that, with a 
smaller size library, buffers which had been continuously sized via the routine min_area_BUF during the optimization process, are now rounded up to a larger buffer because of the wider discretization of the library. As a consequence the area of the tree becomes larger. Moreover, the delay does not change because the use of a larger buffer in a buffering problem like the one posed in the case of routine min_area_BUF (as explained in Section III-C) does not affect the required time at the root of the fanout tree.

Finally, in the remaining problems (e.g. C5315-4 when going from lib-20 to lib-12) when moving from a larger to a smaller size library, both area and delay increase. It turns out that in these cases this effect is due to rounding up a buffer that had been continuously sized during the optimization process via the routine min delay BUF to a larger buffer present in the smaller library. As a result the area increases as does the delay because such a buffer is not optimal for the solution of the original buffering problem and because when a buffer is designed by means of routine min_delay_BUF during the optimization, its delay does propagate to the root of the tree affecting the required time.

The last row of Table II also reports the average percentage variations of the the fanout trees' area and delay. As can be seen, using library lib-12 in place of lib-20 produces fanout trees with $1.1 \%$ more area and $0.2 \%$ more delay. On the other hand if we use lib-8 instead of lib-20, the average area increases by $6.4 \%$ and the average delay by $0.4 \%$. Finally, if we replace lib-20 with lib-4 the increase in area is $5.7 \%$ and the increase in delay is $5.3 \%$.

Therefore, if we accept a modest increase in area and a negligible increase in delay with respect to the optimum case (here represented by library lib-20) we can conclude that library lib-8 satisfies the requirements we have posed for the choice of a discrete size buffer library suitable for employment by our continuous optimization technique. In Table III we also report the distribution of the buffer usage after the optimization of all the fanout problems of Table II for each different discrete size buffer library. As can be seen, because of the increased discretization of the stage ratio, when going from a larger to a smaller size library, the number of used buffers with higher area increases.

\section{B. Individual Fanout Problems}

To provide experimental evidence of the efficiency of the proposed fanout optimization, we have compared the solutions obtained optimizing the fanout trees of the previous section, rounding up buffers against the discrete library lib-8, to those obtained optimizing the same problems with SIS [8]. SIS provides a spectrum of different fanout optimization algorithms, each one based on a different approach: balanced trees, $L T$-trees, combinational merging, two-level trees, top-down traversal. Particularly, while our algorithm has been selected to minimize firstly delay and secondly area (Problem III.1) of the constructed trees, all the other algorithms have been designed to minimize the delay and are then followed by an additional step of area recovery.

Since the fanout algorithms of SIS have not been designed to work with only tapered buffers, for the sake of a fair comparison we have used a different buffer library called lib-std+ (which is a superset of lib-8), containing tapered and non tapered buffers and inverters, when performing the optimization with those algorithms.

The composition of library lib-std+ is reported in Table IV. Here we follow the same notation used in the case of Table I. In this table, library lib-std is composed of all the buffers and inverters that are present in the standard $0.5 \mu \mathrm{m}$ CMOS technology library we have used for mapping the benchmark circuits. As can be seen there are three inverters with different size $(1 \mathrm{x}, 2 \mathrm{x}, 4 \mathrm{x})$ along with tapered and non-tapered buffers. ${ }^{2}$ The minimum inverter $1 \mathrm{x}$ and the tapered buffers $1 \mathrm{x} 1 \mathrm{x}, 1 \mathrm{x} 2 \mathrm{x}, 1 \mathrm{x} 2 \mathrm{x} 4 \mathrm{x}$ are also present in lib-8.

The performance of the trees obtained with our continuous methodology, using library lib-8, has been then compared in the SIS environment with the best result among those achieved by all other SIS fanout optimization techniques using library lib-std+ (i.e. for each fanout problem, all of the SIS fanout optimization algorithms are run and the best result obtained by any of them is reported in the Table). Here, it must be pointed out that

\footnotetext{
${ }^{2}$ We define tapered buffers as those buffers having a minimum inverter as first stage with every successive stage linearly increasing in size with the stage ratio $M$. For instance, $1 \mathrm{x}, 1 \mathrm{x} 1 \mathrm{x}, 1 \mathrm{x} 2 \mathrm{x}, 1 \mathrm{x} 2 \mathrm{x} 4 \mathrm{x}$ are tapered buffers, while $2 \mathrm{x} 4 \mathrm{x}$ and $1 \mathrm{x} 2 \mathrm{x} 8 \mathrm{x}$ are not.
} 


\begin{tabular}{||l||c|c|c|c||}
\hline \hline buffer & lib-20 & lib-12 & lib-8 & lib-4 \\
\hline \hline $1 \times$ & 43 & 43 & 42 & 42 \\
\hline \hline $1 \times 1 \times$ & 22 & 22 & 22 & 23 \\
\hline $1 \times 2 \times$ & 0 & 0 & 0 & \\
\hline $1 \times 2.5 \times$ & 0 & & & \\
\hline $1 \times 3 \times$ & 0 & 0 & & \\
\hline $1 \times 3.5 \times$ & 5 & & & \\
\hline $1 \times 4 \times$ & 12 & 17 & 17 & \\
\hline $1 \times 4.5 \times$ & 8 & & & \\
\hline $1 \times 5 \times$ & 7 & 15 & & \\
\hline $1 \times 5.5 \times$ & 7 & & & \\
\hline $1 \times 6 \times$ & 15 & 22 & 36 & 53 \\
\hline $1 \times 2 \times 2^{2} \times$ & 0 & 0 & 1 & \\
\hline $1 \times 2.5 \times 2.5^{2} \times$ & 1 & & & \\
\hline $1 \times 3 \times 3^{2} \times$ & 1 & 2 & & \\
\hline $1 \times 3.5 \times 3.5^{2} \times$ & 2 & & & \\
\hline $1 \times 4 \times 4^{2} \times$ & 8 & 10 & 12 & 12 \\
\hline $1 \times 2 \times 2^{2} \times 2^{3} \times$ & 0 & 0 & 0 & \\
\hline $1 \times 2.5 \times 2.5^{2} \times 2.5^{3} \times$ & 1 & & & \\
\hline $1 \times 3 \times 3^{2} \times 3^{3} \times$ & 0 & 1 & & \\
\hline $1 \times 3.5 \times 3.5^{2} \times 3.5^{3} \times$ & 0 & & & \\
\hline $1 \times 4 \times 4^{2} \times 4^{3} \times$ & 0 & 0 & 1 & 1 \\
\hline \hline
\end{tabular}

TABLE III

BUFFER USAGE DISTRIBUTION OF THE OPTIMIZED FANOUT TREES USING FOUR DIFFERENT DISCRETE SIZE BUFFER LIBRARIES.

all of the fanout optimization algorithms in SIS have been modified to take the DMSlib format. The results are reported in Table V. In this table the continuous field reports the results obtained by our optimization algorithm (the same as those of Table II in the field lib-8) while the sis field reports the best results obtained with the other algorithms. In both fields the terms area and delay have the same meaning as in Table II. Furthermore, column $g$ reports the number of gates (inverters and buffers) the trees are composed of, and the cpu column reports the user time in seconds needed for the computation. Finally, the last two columns under field $\Delta \%$ report the percentage variation of delay and area under the continuous field with respect to the corresponding values reported in the sis field. As can be seen, due to the wider tree search space and the adoption of routines min_delay_BUF and min_area_BUF in all cases the tree_selection algorithm generates fanout trees with a consistently lower area, the average reduction against the other algorithms being $60 \%$. As for the delay, in the majority of the cases our algorithm performs better than the others with an average reduction of $10 \%$. Here it must be pointed out that the use of lib-std+ for the SIS algorithms puts our algorithm at a disadvantage since it only uses lib-8 which is only a subset of lib-std+. Regarding the execution time, because of its low complexity, in almost all cases the computation time of our algorithm is much lower than the corresponding time needed by the other algorithms. In this experiment, the typical execution time of routines min_delay_BUF and min_area_BUF, which are in the most nested inner loop of our dynamic programming algorithm, were $0.35 \mathrm{~ms}$ and $3.73 \mathrm{~ms}$, respectively. Finally, in order to verify the accuracy of the adopted delay model, each fanout tree has been simulated with the SPICE 


\begin{tabular}{||l|c||c|c|c||}
\hline \hline buffer & area & lib-8 & lib-std & lib-std+ \\
\hline \hline $1 \mathrm{x}$ & 687.5 & $\bullet$ & $\bullet$ & $\bullet$ \\
\hline \hline $2 \mathrm{x}$ & 770.0 & & $\bullet$ & $\bullet$ \\
\hline $4 \mathrm{x}$ & 852.5 & & $\bullet$ & $\bullet$ \\
\hline $1 \mathrm{x} 1 \mathrm{x}$ & 852.5 & $\bullet$ & $\bullet$ & $\bullet$ \\
\hline $1 \mathrm{x} 2 \mathrm{x}$ & 935.0 & $\bullet$ & $\bullet$ & $\bullet$ \\
\hline $1 \mathrm{x} 4 \mathrm{x}$ & 1017.5 & $\bullet$ & & $\bullet$ \\
\hline $1 \mathrm{x} 6 \mathrm{x}$ & 1100.0 & $\bullet$ & & $\bullet$ \\
\hline $2 \mathrm{x} 4 \mathrm{x}$ & 1100.0 & & $\bullet$ & $\bullet$ \\
\hline $2 \mathrm{x} 8 \mathrm{x}$ & 1265.0 & & $\bullet$ & $\bullet$ \\
\hline $4 \mathrm{x} 16 \mathrm{x}$ & 1677.5 & & $\bullet$ & $\bullet$ \\
\hline $1 \mathrm{x} 2 \mathrm{x} 4 \mathrm{x}$ & 1265.0 & $\bullet$ & $\bullet$ & $\bullet$ \\
\hline $1 \mathrm{x} 2 \mathrm{x} 8 \mathrm{x}$ & 1430.0 & & $\bullet$ & $\bullet$ \\
\hline $2 \mathrm{x} 4 \mathrm{x} 16 \mathrm{x}$ & 1925.0 & & $\bullet$ & $\bullet$ \\
\hline $1 \mathrm{x} 4 \mathrm{x} 16 \mathrm{x}$ & 1842.5 & $\bullet$ & & $\bullet$ \\
\hline $1 \mathrm{x} 2 \mathrm{x} 4 \mathrm{x} 8 \mathrm{x}$ & 1760.0 & $\bullet$ & & $\bullet$ \\
\hline $1 \mathrm{x} 4 \mathrm{x} 16 \mathrm{x} 64 \mathrm{x}$ & 4647.5 & $\bullet$ & & $\bullet$ \\
\hline \hline
\end{tabular}

TABLE IV

COMPOSITION OF THE DISCRETE SIZE BUFFER LIBRARIES LIB-STD AND LIB-STD+. LIBRARY LIB-STD+ IS THE UNION OF LIBRARIES LIB-STD AND LIB-8.

program. The average error on the calculation of signal delay and slopes at the sinks was $5 \%$.

\section{Global Fanout Optimization}

After testing the algorithm for a significant number of typical fanout problems, the optimization algorithm is now applied to entire circuits and its performance compared with those of the other techniques mentioned in Section IV-B. To this purpose, the algorithm has been implemented in the SIS environment. Here, for each different fanout algorithm, the procedure used for the global optimization of a circuit is that presented in [9]. With this methodology every node is visited in topological order and when a fanout problem is encountered, a fanout tree which is in turn constructed by a selected algorithm is introduced. In [5], this procedure is shown to be optimal with respect to delay minimization.

In Table VI, the results of the global fanout optimization performed for minimum delay on a variety of benchmark circuits are reported. The mapping field reports the delay and area of the circuits after the execution of the technology mapping step for minimum delay. The second field reports the results of the best fanout optimization obtained from the spectrum of algorithms available in SIS using the discrete buffer library lib-std+. The third field reports the results obtained by optimizing the circuits with the proposed continuous methodology using the discrete buffer library lib-8. Finally, the last two columns report the performance comparison between the two approaches. Here, it must be pointed out that while the first approach selects, for each node, the best solution among those produced by each of the considered algorithms present in SIS, the continuous approach, in all cases, performs the optimization in the same way by means of the tree_selection algorithm. Moreover, it is worth noting that our algorithm is put at a disadvantage with respect to the other algorithms since the library of buffers that it uses for its optimization process (lib-8) is only a subset of the library lib-std+ used by the other algorithms. 


\begin{tabular}{||c|c||r|r|r|r|r|r|r|r|r|r||}
\hline \hline \multicolumn{2}{||c||}{ problem } & \multicolumn{9}{|c|}{ sis } & \multicolumn{6}{c|}{ continuous } & \multicolumn{2}{|c|}{$\Delta \%$} \\
\hline circuit & sinks & $g$ & delay & area & cpu & $g$ & delay & area & cpu & delay & area \\
\hline \hline C1355-1 & 8 & 7 & 0.44 & 6050 & 1.6 & 4 & 0.44 & 3410 & 0.1 & 0 & -44 \\
\hline C1355-2 & 9 & 6 & 0.58 & 5362 & 1.7 & 5 & 0.47 & 3932 & 0.1 & -19 & -27 \\
\hline C1355-3 & 9 & 4 & 0.55 & 4072 & 1.9 & 4 & 0.53 & 3740 & 0.1 & -4 & -8 \\
\hline C1355-4 & 13 & 5 & 0.36 & 4180 & 4.3 & 1 & 0.36 & 1018 & 0.0 & 0 & -76 \\
\hline C3540-1 & 12 & 11 & 0.41 & 9295 & 2.8 & 6 & 0.41 & 4455 & 0.2 & 0 & -52 \\
\hline C3540-2 & 35 & 18 & 0.33 & 17078 & 32.7 & 3 & 0.24 & 6600 & 1.0 & -27 & -61 \\
\hline C3540-3 & 21 & 10 & 0.48 & 8442 & 9.5 & 4 & 0.50 & 3740 & 0.2 & 4 & -56 \\
\hline C3540-4 & 72 & 25 & 0.57 & 22965 & 76.1 & 6 & 0.20 & 7012 & 6.1 & -67 & -69 \\
\hline C432-1 & 16 & 14 & 0.28 & 13502 & 6.8 & 2 & 0.23 & 1952 & 0.7 & -18 & -86 \\
\hline C432-2 & 6 & 3 & 0.24 & 3300 & 1.3 & 1 & 0.21 & 852 & 0.0 & -12 & -74 \\
\hline C432-3 & 16 & 13 & 0.27 & 11165 & 6.1 & 3 & 0.30 & 2888 & 0.3 & 11 & -74 \\
\hline C432-4 & 10 & 10 & 0.26 & 9624 & 1.6 & 3 & 0.16 & 2558 & 0.1 & -38 & -73 \\
\hline C5315-1 & 49 & 21 & 0.45 & 19635 & 38.6 & 11 & 0.49 & 10532 & 1.5 & 9 & -46 \\
\hline C5315-2 & 12 & 8 & 0.28 & 6737 & 3.5 & 4 & 0.30 & 3080 & 0.2 & 7 & -54 \\
\hline C5315-3 & 21 & 12 & 0.38 & 10807 & 6.3 & 9 & 0.40 & 7178 & 0.3 & 5 & -34 \\
\hline C5315-4 & 50 & 27 & 0.47 & 27885 & 49.1 & 11 & 0.52 & 10450 & 3.3 & 11 & -63 \\
\hline C6288-1 & 16 & 15 & 0.16 & 15427 & 6.9 & 1 & 0.17 & 1100 & 1.1 & 6 & -93 \\
\hline C6288-2 & 21 & 20 & 0.16 & 20845 & 11.7 & 1 & 0.17 & 1100 & 2.2 & 6 & -95 \\
\hline C6288-3 & 60 & 17 & 0.74 & 14905 & 61.1 & 10 & 0.29 & 8938 & 9.0 & -61 & -40 \\
\hline C6288-4 & 50 & 30 & 0.16 & 30195 & 48.2 & 1 & 0.17 & 1100 & 3.2 & 6 & -96 \\
\hline C7552-1 & \multicolumn{1}{|c||}{483} & 41 & 0.73 & 47000 & 1439.8 & 31 & 0.56 & 38802 & 15.1 & -23 & -18 \\
\hline C7552-2 & 12 & 7 & 0.26 & 5885 & 3.1 & 2 & 0.26 & 1788 & 0.4 & 0 & -70 \\
\hline C7552-3 & 16 & 11 & 0.29 & 9872 & 4.5 & 6 & 0.23 & 4620 & 0.5 & -21 & -53 \\
\hline C7552-4 & 23 & 15 & 0.21 & 12292 & 10.8 & 2 & 0.18 & 1788 & 0.7 & -14 & -85 \\
\hline \hline average & & & & & & & & & & -10 & -60 \\
\hline \hline
\end{tabular}

TABLE V

RESUlTS FOR DELAY OPTIMIZED FANOUT TREES. FOR EACH TREE, $g$ REPRESENTS THE NUMBER OF GATES (BUFFERS AND INVERTERS), DELAY IS THE DIFFERENCE IN NANOSECONDS BETWEEN THE REQUIRED TIME AT THE MOST CRITICAL SINK AND THE REQUIRED TIME AT THE ROOT, AND CPU IS THE RUN-TIME IN SECONDS ON A SUN-SPARC 20. THE TREE AREA IS GIVEN IN $\mu \mathrm{m}^{2}$.

As can be seen, with the continuous approach every circuit is optimized in shorter time and the resulting implementation has on average lower delay and lower area. Particularly, the typical reduction is $4 \%$ in delay and $34 \%$ in area, while the computation time is typically one order of magnitude lower. It is worth noting that these figures are consistent with those reported in Table V considering that on average, up to $30 \%$ of the nodes of a circuit are typically optimized due to their large fanout count. Regarding the execution of the global fanout optimization performed in the SIS environment, an important consideration must be made. In particular, while all of the SIS algorithms have been run with the -FG options, the tree_selection has been executed with only the -F option since its gate selection process is implicitly optimal. On the other hand, in both cases the area recovery option -A has been excluded because such a step does not take into account the slope of the signals of the internal nodes introducing an unacceptable additional delay in the optimized circuit. Particularly, it has been observed that running the global fan-out optimization with the option -A, for all algorithms, the resulting circuits have lower area but also delay typically $15 \%$ higher than reported in either Table VI or Table VII. In the last experiment, we have performed again the fanout optimization of the benchmark circuits as in the previous case, but 
this time making available to all algorithms only those buffers that were originally included in the standard technology library used for the mapping of the circuits (i.e. those in the discrete size library lib-std). In this case our algorithm was only able to use the tapered buffers included in library lib-std, that is $1 \mathrm{x}, 1 \mathrm{x} 1 \mathrm{x}, 1 \mathrm{x} 2 \mathrm{x}$ and $1 \mathrm{x} 2 \mathrm{x} 4 \mathrm{x}$. The corresponding results are reported in Table VII. As can be seen, all algorithms have inferior performance in terms of delay with respect to the results of Table VI. Nonetheless, the average improvements of our algorithm versus the other algorithms remain the same.

\section{CONCLUSiOn AND Future Work}

In this paper we have presented a new methodology for the solution of the fanout problem based on a continuous delay optimization technique. An accurate transistor-level delay model is used to design delay and area optimized buffers that perfectly fit the slacks between the leaves of the fanout tree they set up, resulting in consistent area savings. Our approach is particularly effective for circuits developed with submicron CMOS processes where special care must be taken in the evaluation of delay times and signal slope effects. A polynomial time algorithm which uses dynamic programming for the selection of the best possible fanout tree, has also been presented. The high accuracy of its delay model, the independence from the technology in use, the wide tree search space, and the fast run-time make the algorithm very convenient to be used in CAD tools for the automatic synthesis of digital circuits.

One limitation of all the fanout algorithms that have been considered so far is that they do not consider the effect of the delay introduced by the interconnections that propagate a signal from the source to its destinations. If such an effect is modeled assuming that each interconnect can be substituted by a corresponding lumped capacitance, the solution to this problem is straightforward and only requires the addition of such a capacitance in the load of the corresponding sink. On the other hand, when deep submicron technologies are used, in several cases the resistance of the interconnections cannot be neglected any more and new optimization strategies must be adopted to comprehend this effect. One of the most promising of such strategies is the unification-based approach for which new algorithms are developed to solve diverse optimization steps at the same time. Following this strategy we are planning to extend our work to the simultaneous solution of routing tree construction and fanout optimization problems, which has already been accomplished in [10] in the case of the $L T$-Tree for fanout structure and the P-Tree for routing structure.

\section{REFERENCES}

[1] T. Aoki, M. Murakata, T. Mitsuhashi, and N. Goto, "Fanout-tree restructuring algorithm for post-placement timing optimization," in ASP-DAC, Aug. 1995, pp. 417-422.

[2] C. L. Berman, J. L. Carter, and K. F. Day, "The fanout problem: From theory to practice," in Advanced Research on VLSI: Proc. of the 1989 Decennial Caltech Conference, C. L. Seitz, Ed. MIT press, Mar. 1989, pp. 69-99.

[3] M. C. Golumbic, "Combinatorial merging," IEEE Transactions on Computers, vol. 25, pp. 1164-1167, Nov. 1976.

[4] K. J. Singh and A. Sangiovanni-Vincentelli, "A heuristic algorithm for the fanout problem," in Proceedings of the 27th Design Automation Conference, June 1990, pp. 357-360.

[5] H. Touati, Performance-oriented technology mapping, Ph.D. thesis, University of California, Berkeley, Nov. 1990, Technical Report UCB/ERL M90/109.

[6] H. Vaishnav and M. Pedram, "Routability-driven fanout optimization," in Proceedings of the 30th Design Automation Conference, June 1993, pp. 230-235.

[7] P. Cocchini, G. Piccinini, and M. Zamboni, "A comprehensive submicrometer MOST delay model and its application to CMOS buffers," IEEE J. Solid-State Circuits, vol. 32, no. 8, pp. 1254-1262, Aug. 1997.

[8] E. M. Sentovich, K. J. Singh, C. Moon, H. Savoj, R. K. Brayton, and A. Sangiovanni-Vincentelli, "Sequential circuit design using synthesis and optimization," in VLSI in Computers \& Processors: Proc. of the 1992 IEEE International Conference on Computer Design, R. Werner, Ed. IEEE Computer Society Press, Oct. 1992, pp. 328-333.

[9] H. J. Hoover, M. M. Klawe, and N. J. Pippinger, "Bouding fan-out in logical networks," Journal of the Association for Computing Machinery, vol. 31, no. 1, pp. 13-18, Jan. 1984.

[10] A. Salek, J. Lou, and M. Pedram, “A simultaneous routing tree construction and fanout optimization algorithm," in IEEE/ACM International Conference on CAD, Nov. 1998, pp. 625-630.

[11] G. Massobrio and P. Antognetti, Semiconductor Modeling with SPICE, McGrow-Hill, 1993. 
[12] S. M. Sze, Physics of Semiconductor Devices, Wiley, 1981.

[13] Yannis P. Tsividis, Operation and Modeling of the MOS transistor, McGrow-Hill, 1987.

\section{APPENDIX}

\section{Delay Model}

We refer here to the calculation of the propagation time $t_{p f}$ of the inverter depicted in Figure 1, although the results can be applied to the evaluation of any other delay time. For a falling output transition, $C_{F F}$, the feedforward capacitance, is equal to the overlap capacitance of the NMOS transistor $T_{1}$ plus the capacitance introduced by the PMOS transistor $T_{2}$. At $t=0, C_{L}$ is charged at $V_{D D}$ and the input voltage is zero. For $t>0$ the input voltage $V_{I}$ increases as $V_{I}=K_{I} t$ until it reaches $V_{D D}$ and then remains constant, while $V_{O}$, after an initial increase, decreases to reach a switching voltage $V_{S}=V_{D D} / 2$. During the transition of $V_{O}$ from $V_{D D}$ to $V_{S}$, depending on the slope $K_{I}$ of the input voltage, five different regions of operation of the NMOS transistor can be distinguished as shown in Fig. 7.

In region 0 , for $0<t<t_{0}$, the transistor is off and $\Delta_{0}=t_{0}$ is the time that $V_{I}$ needs to switch the transistor on while $C_{L}$ is being charged by the current $I_{F F}$ due to the high slew rate of the input voltage, so that $V_{O}$ increases.

In region 1 , for $t_{0}<t<t_{1}, T_{1}$ is in saturation while the input voltage $V_{I}$ is still increasing reaching for $V_{D D}$. If at the time $t_{D D}=V_{D D} / K_{I}$, the voltage $V_{I}$ reaches $V_{D D}$ when the transistor is still in saturation, then it enters region 2, otherwise if the transistor goes in linearity while $V_{I}$ is still less than $V_{D D}$, region 3 is entered. In both cases $\Delta_{1}=t_{1}-t_{0}$ is the time needed for the transition.

In region 2 , for $t_{1}<t<t_{2}, T_{1}$ is in saturation and $V_{I}$ is constant and equal to $V_{D D}$. After the time $\Delta_{2}=$ $t_{2}-t_{1}$, with the decrease of $V_{O}$, the transistor leaves its own drain current saturation region to enter region 4.

For $t_{1}<t<t_{3}, T_{1}$ works in region 3 , where the transistor is in linearity and the input voltage has not yet reached the supply voltage. In this situation, if $V_{O}$ reaches $V_{S}$ during the time $\Delta_{3}=t_{3}-t_{1}$, then the overall propagation time $t_{p f}$ is equal to $t_{p f}=t_{3}-t_{D D} / 2=\Delta_{0}+\Delta_{1}+\Delta_{3}-t_{D D} / 2$. On the contrary, if $V_{I}$ reaches $V_{D D}$ while the output voltage is still less than $V_{S}$, then region 4 is entered.

In region $4, T_{1}$ is in linearity and $V_{I}$ is stuck at $V_{D D}$. The time needed by $V_{O}$ to reach $V_{S}$ is either $\Delta_{4}=t_{4}-t_{2}$ or $\Delta_{4}=t_{4}-t_{3}$ and the overall propagation time becomes $t_{p f}=t_{4}-t_{D D} / 2=\Delta_{0}+\Delta_{1}+\Delta_{2}+\Delta_{4}-t_{D D} / 2$ or $t_{p f}=t_{4}-t_{D D} / 2=\Delta_{0}+\Delta_{1}+\Delta_{3}+\Delta_{4}-t_{D D} / 2$, respectively.

The output response of a minimum inverter, loaded by another minimum inverter and driven by a trapezoidal shape input voltage, for a $0.7 \mu \mathrm{m}$ CMOS reference technology is reported in Figure 8 along with the result of a corresponding SPICE simulation. Here, a dashed line indicates the way the different regions of operation are crossed during the whole output transition. For a wide range of input voltage slopes and capacitive loads, the average error on the calculation of the propagation time is $3 \%$.

\section{BUFFER OPTIMIZATION}

Like other works based on tapered buffers, the optimization methodology we use relies on the assumption that the overall propagation delay of a speed optimized buffer is uniformly distributed along the structure. The scheme of such buffers, that we have already introduced in Section II-B, is shown in Figure 2. We refer here to the definitions of delays and slopes given in the previous sections. For a more detailed treatment, the reader is referred to [7].

A thorough analysis of the circuit shows that under the above mentioned assumption, every stage $i \in\{1, \ldots, N\}$ has exactly the same behavior in terms of propagation delay $t_{p_{i}}\left(t_{p_{i}}=t_{p r_{i}}=t_{p f_{i}}\right)$ and output slopes $k_{r_{O_{i}}}$ and $k_{f_{O_{i}}}$, provided that the buffer input signal has rising and falling slopes equal to those of the output, that is $k_{r_{O_{N}}}=k_{r_{I_{1}}}$ and $k_{f_{O_{N}}}=k_{f_{I_{1}}}$. Therefore, to predict the timing of the whole circuit, it is sufficient to model only one stage. To this purpose, the inverter delay model introduced in Section II is implemented in a one-step characterization algorithm, which gives all the needed relations between the tapering factor $M$, the width ratio $u_{i}$, and the output delays and slopes of the stage. This is done by means of four different fitted equations 


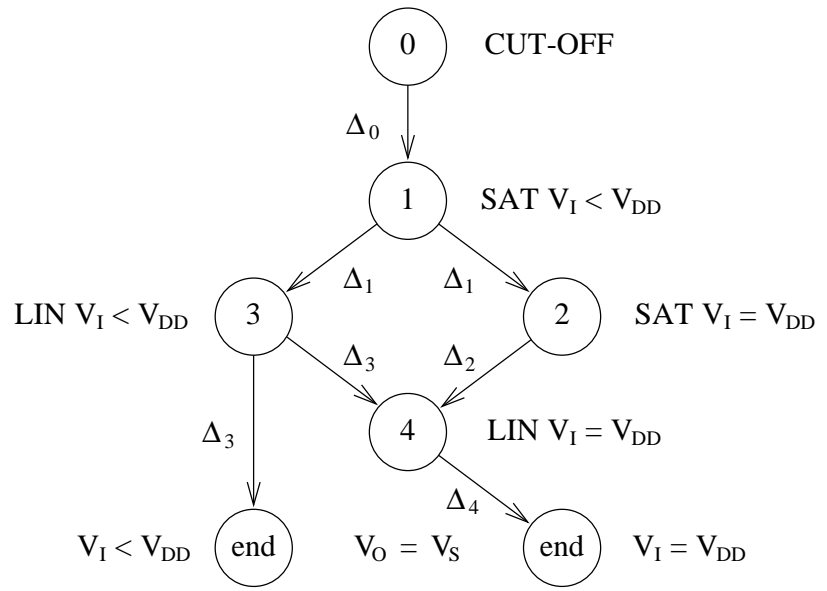

Fig. 7. MOST regions of operation.

$$
\begin{array}{cc}
t_{p_{i}}=a_{1}+a_{2} M & u_{i}=a_{3}+a_{4} \ln (M) \\
k_{r_{O_{i}}}=\left(a_{5}+a_{6} M\right)^{-1} & k_{f_{O_{i}}}=\left(a_{7}+a_{8} M\right)^{-1}
\end{array}
$$

Fig. 8. Output response of a minimum inverter.

where parameters $a_{1}, \cdots, a_{8}$ are the results of the characterization. In this way, a fast and accurate model of the basic stage is available for the optimization of the buffer delay.

The overall propagation time $t_{p}$ of the buffer is $N$ times that of a single stage $t_{p_{i}}$. Thus, for uniformity of stage delays, we must have

$$
t_{p}=N t_{p_{i}}=\frac{\ln \left(C_{L} / C_{1}\right)}{\ln (M)}\left(a_{1}+a_{2} M\right)
$$

where $C_{L}$ and $C_{1}$ are the load capacitance and the stage input capacitance, respectively. By imposing the derivative of $t_{p}$ with respect to $M$ to be equal to zero, it is possible to find an expression for the optimized value of $M$ which corresponds to the minimum overall propagation delay of the chain.

$$
M_{o p t}=\exp \left(1+\frac{a_{1}}{a_{2} M_{o p t}}\right)
$$

Equation (8) can be easily solved by successive iterations considering that $M_{\text {opt }}$ must be greater than $e$. The number of stages to be implemented in the buffer is then

$$
N=\left\lfloor K_{a s} \frac{\ln \left(C_{L} / C_{1}\right)}{\ln \left(M_{o p t}\right)}+0.5\right\rfloor
$$

The coefficient $K_{a s}$, which is usually taken to be close to unity, is introduced to decrease the number of stages in order to reduce the total amount of area used. By adjusting $K_{a s}$, a good compromise between area and speed can be achieved.

After the determination of $\mathrm{N}$, the optimum tapering factor must be recalculated, that is

$$
M_{\text {opt }}=\left(\frac{C_{L}}{C_{1}}\right)^{\frac{1}{N}}
$$




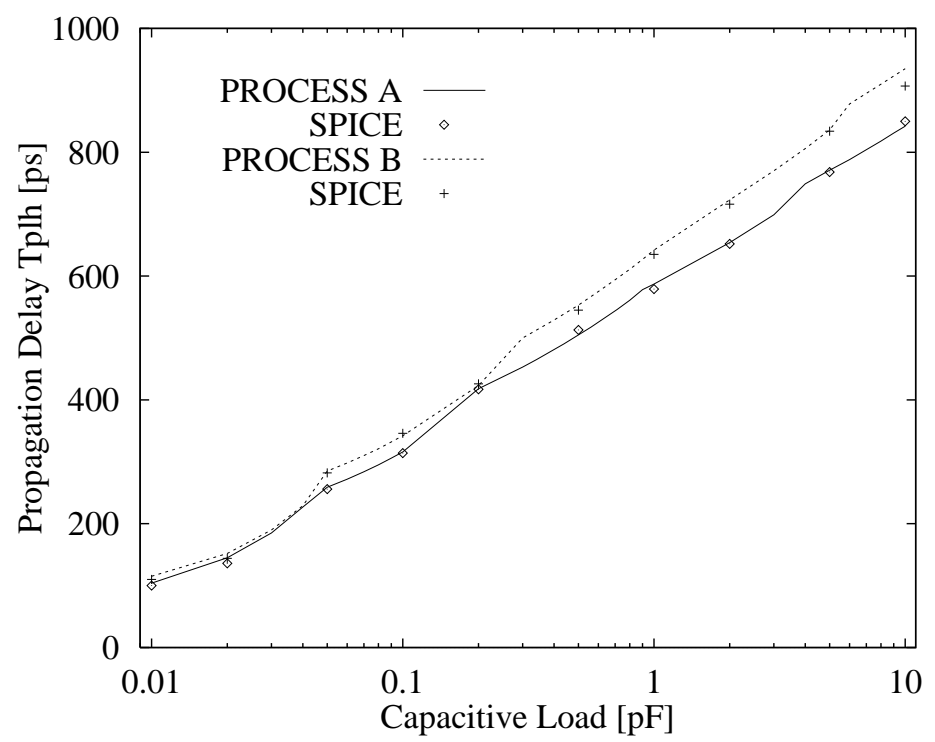

Fig. 9. Delay optimized CMOS tapered buffers designed for two different $0.7 \mu \mathrm{m}$ technologies.

At this point, the width ratio $u_{i}$ of each stage which provides a symmetrical output response of the buffer, and the overall propagation time $t_{p}$ can be found by substituting $M_{\text {opt }}$ in the equations previously introduced, so that

$$
u_{i}=a_{3}+a_{4} \ln \left(M_{o p t}\right)
$$

and

$$
t_{p}=N\left(a_{1}+a_{2} M_{o p t}\right)
$$

A design example carried out for two different $0.7 \mu \mathrm{m}$ CMOS processes, namely process $\mathrm{A}$ and $\mathrm{B}$, is reported in Figure 9. In the same figure, the results of several SPICE simulation are also reported to test the accuracy of the calculated delay. As can be seen, for both processes A and B, the designed buffers show high precision for a wide range of load capacitances. Particularly, with $C_{L}$ from $50 \mathrm{fF}$ to $10 \mathrm{pF}$ the agreement with SPICE simulations is better than $3 \%$, while with smaller values of $C_{L}$ the accuracy is about $6 \%$. 


\begin{tabular}{|c|c|c|c|c|c|c|c|c|c|c|c|c|c|c|}
\hline & \multicolumn{2}{|c|}{ mapping } & \multicolumn{5}{|c|}{ sis } & \multicolumn{5}{|c|}{ continuous } & \multicolumn{2}{|c|}{$\Delta \%$} \\
\hline circuit & delay & area & delay & $\Delta \%$ & area & $\Delta \%$ & сpu & delay & $\Delta \%$ & area & $\Delta \%$ & сpu & delay & area \\
\hline 9symml & 5.73 & 76 & 4.94 & -13.8 & 189 & 148.6 & 51 & 4.47 & -22.0 & 116 & 51.9 & 3 & -9.5 & -38.9 \\
\hline C1355 & 6.28 & 258 & 6.12 & -2.6 & 494 & 91.0 & 65 & 6.01 & -4.3 & 344 & 33.3 & 2 & -1.8 & -30.2 \\
\hline C2670 & 8.38 & 351 & 6.71 & -19.9 & 706 & 101.4 & 108 & 6.61 & -21.1 & 495 & 41.1 & 4 & -1.5 & -30.0 \\
\hline C3540 & 16.36 & 524 & 12.47 & -23.8 & 1140 & 117.6 & 272 & 12.45 & -23.9 & 760 & 45.0 & 16 & -0.2 & -33.4 \\
\hline C5315 & 10.38 & 774 & 8.21 & -20.9 & 1611 & 108.1 & 299 & 8.17 & -21.3 & 1106 & 42.8 & 11 & -0.5 & -31.4 \\
\hline C6288 & 32.48 & 1514 & 27.46 & -15.4 & 3325 & 119.7 & 395 & 27.31 & -15.9 & 2123 & 40.2 & 30 & -0.5 & -36.2 \\
\hline C7552 & 23.52 & 1005 & 13.70 & -41.7 & 2152 & 114.2 & 582 & 10.95 & -53.4 & 1456 & 45.0 & 22 & -20.1 & -32.3 \\
\hline alu2 & 9.47 & 165 & 7.49 & -20.9 & 382 & 132.0 & 68 & 7.62 & -19.5 & 247 & 50.1 & 4 & 1.7 & -35.3 \\
\hline alu4 & 12.24 & 307 & 10.55 & -13.8 & 706 & 130.1 & 131 & 9.66 & -21.1 & 463 & $\overline{50.9}$ & 8 & -8.4 & -34.4 \\
\hline apex6 & 7.60 & 323 & 5.18 & -31.8 & 723 & 123.9 & 104 & 5.11 & -32.8 & 460 & 42.5 & 3 & -1.4 & -36.3 \\
\hline apex7 & 5.43 & 117 & 3.69 & -32.1 & 244 & 109.4 & 58 & 3.55 & -34.6 & 171 & 46.6 & 1 & -3.8 & -30.0 \\
\hline comp & 3.79 & 61 & 3.44 & -9.3 & 112 & 83.9 & 8 & 3.51 & -7.4 & 79 & 30.0 & 0 & 2.0 & -29.3 \\
\hline dalu & 19.11 & 544 & 13.43 & -29.7 & 1228 & 125.9 & 224 & 13.12 & -31.3 & 780 & 43.5 & 17 & -2.3 & -36.5 \\
\hline k2 & 8.93 & 511 & 7.37 & -17.5 & 1128 & 120.6 & 156 & 7.18 & -19.6 & 793 & 55.2 & 7 & -2.6 & -29.6 \\
\hline misex3 & 8.54 & $\overline{249}$ & 6.20 & -27.4 & 612 & 146.4 & 177 & 5.85 & -31.5 & 385 & 54.9 & 13 & -5.6 & -37.1 \\
\hline rot & 8.17 & 320 & 6.09 & -25.5 & 711 & 122.4 & 88 & 6.22 & -23.9 & 468 & 46.4 & 4 & 2.1 & -34.1 \\
\hline $\mathrm{x} 2$ & 2.51 & 34 & 2.33 & -7.3 & 69 & 100.7 & 12 & 2.07 & -17.6 & 44 & 28.9 & 0 & -11.2 & -35.8 \\
\hline $\mathrm{x} 4$ & 6.71 & 280 & 3.22 & -52.0 & 563 & 100.8 & 267 & 3.17 & -52.7 & 355 & 26.6 & 3 & -1.6 & $\begin{array}{l}-36.9 \\
\end{array}$ \\
\hline average & & & & -22.5 & & 116.5 & & & -25.2 & & 43.1 & & -3.6 & -33.8 \\
\hline
\end{tabular}

TABLE VI

RESULTS FOR MAXIMUM SPEED FAN-OUT OPTIMIZATION APPLIED TO ENTIRE CIRCUITS USING THE DISCRETE SIZE BUFFER LIBRARY LIB-8 FOR THE continuous APPROACH AND LIBRARY LIB-STD+ FOR THE Sis ALGORITHMS: DELAY IS GIVEN IN NANOSECONDS, AREA IS GIVEN IN $10^{3} \mu \mathrm{m}^{2}$, AND CPU IS THE RUN-TIME IN SECONDS ON A

SUN-ULTRA 2. 


\begin{tabular}{|c|c|c|c|c|c|c|c|c|c|c|c|c|c|c|}
\hline & \multicolumn{2}{|c|}{ mapping } & \multicolumn{5}{|c|}{ sis } & \multicolumn{5}{|c|}{ continuous } & \multicolumn{2}{|c|}{$\Delta \%$} \\
\hline circuit & delay & area & delay & $\Delta \%$ & area & $\Delta \%$ & сpu & delay & $\Delta \%$ & area & $\Delta \%$ & $\mathrm{cpu}$ & delay & area \\
\hline 9symml & $\overline{\overline{5.73}}$ & 76 & 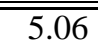 & -11.7 & 186 & $\overline{145.2}$ & 30 & 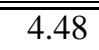 & -21.9 & $\overline{115}$ & $\overline{\overline{551.7}}$ & $\overline{\bar{z}}$ & -11.5 & -38.1 \\
\hline C1355 & 6.28 & 258 & 6.12 & -2.6 & 494 & 91.0 & 39 & 6.01 & -4.3 & 344 & 33.3 & 2 & -1.8 & -30.2 \\
\hline $\mathrm{C} 2670$ & 8.38 & 351 & 6.71 & -19.9 & 706 & 101.4 & 67 & 6.64 & -20.7 & 490 & 39.8 & 4 & -1.0 & -30.6 \\
\hline C3540 & 16.36 & 524 & 12.44 & -23.9 & 1142 & 118.0 & 160 & 12.81 & -21.7 & 757 & 44.5 & 17 & 3.0 & -33.7 \\
\hline C5315 & 10.38 & 774 & 8.54 & -17.7 & 1616 & 108.7 & 175 & 8.33 & -19.7 & 1088 & 40.5 & 12 & -2.5 & -32.7 \\
\hline C6288 & 32.48 & 1514 & 27.46 & $\begin{array}{l}-15.4 \\
\end{array}$ & 3325 & 119.7 & 239 & 27.63 & $\begin{array}{l}-14.9 \\
\end{array}$ & 2123 & 40.3 & 30 & 0.6 & -36.1 \\
\hline C7552 & 23.52 & 1005 & 16.00 & -32.0 & 2162 & 115.2 & 343 & 11.16 & -52.5 & 1453 & 44.6 & 22 & -30.2 & -32.8 \\
\hline alu2 & 9.47 & 165 & 7.49 & -20.9 & 382 & 132.0 & 43 & 7.62 & -19.5 & 247 & 50.0 & 4 & 1.7 & -35.3 \\
\hline alu4 & 12.24 & 307 & 10.55 & -13.8 & 706 & 130.1 & 79 & 9.69 & -20.8 & 462 & 50.7 & 8 & -8.2 & -34.5 \\
\hline apex6 & 7.60 & 323 & 5.18 & -31.8 & 723 & 123.9 & 70 & 5.11 & -32.8 & 458 & 41.9 & 3 & -1.4 & -36.6 \\
\hline apex7 & 5.43 & 117 & 3.69 & -32.1 & 245 & 110.3 & 36 & 3.56 & -34.5 & 171 & 46.3 & 1 & -3.5 & -30.4 \\
\hline comp & 3.79 & 61 & 3.44 & -9.3 & 112 & 83.9 & 5 & 3.51 & -7.4 & 79 & 30.0 & 0 & 2.0 & -29.3 \\
\hline dalu & 19.11 & 544 & 13.37 & -30.0 & 1227 & 125.7 & 136 & 13.02 & -31.9 & 776 & 42.8 & 17 & -2.6 & -36.7 \\
\hline $\mathrm{k} 2$ & 8.93 & 511 & 7.33 & -17.9 & 1134 & 121.9 & 93 & 7.21 & -19.3 & 792 & 55.0 & 7 & -1.6 & -30.1 \\
\hline misex 3 & 8.54 & 249 & 6.20 & -27.4 & 612 & 146.4 & 106 & 5.86 & -31.4 & 388 & 56.1 & 12 & -5.5 & -36.7 \\
\hline rot & 8.17 & 320 & 6.09 & -25.5 & 711 & 122.4 & 54 & 6.36 & -22.2 & 465 & 45.6 & 5 & 4.4 & -34.5 \\
\hline $\mathrm{x} 2$ & 2.51 & 34 & 2.33 & -7.3 & 69 & 100.7 & 7 & 2.11 & -16.0 & 44 & 28.6 & 0 & -9.4 & -35.9 \\
\hline $\mathrm{x} 4$ & 6.71 & $\overline{280}$ & 3.22 & -52.0 & 563 & 100.8 & 159 & 3.24 & -51.7 & 352 & 25.5 & 3 & 0.6 & -37.5 \\
\hline average & & & & -21.7 & & 116.5 & & & -24.6 & & 42.6 & & -3.7 & -34.0 \\
\hline
\end{tabular}

TABLE VII

RESULTS FOR MAXIMUM SPEED FAN-OUT OPTIMIZATION APPLIED TO ENTIRE CIRCUITS USING THE DISCRETE SIZE BUFFER LIBRARY LIB-STD FOR ALL ALGORITHMS: DELAY IS GIVEN IN NANOSECONDS, AREA IS GIVEN IN $10^{\beta} \mu \mathrm{m}^{2}$, AND CPU IS THE RUN-TIME IN SECONDS ON A SUN-ULTRA 2. 